medRxiv preprint doi: https://doi.org/10.1101/2021.02.23.21252221; this version posted February 26, 2021. The copyright holder for this preprint (which was not certified by peer review) is the author/funder, who has granted medRxiv a license to display the preprint in perpetuity.

All rights reserved. No reuse allowed without permission.

\title{
TITLE: \\ Microbial signatures in the lower airways of mechanically ventilated COVID19 patients associated with poor clinical outcome
}

Imran Sulaiman ${ }^{1,2}$, Matthew Chung ${ }^{3}$, Luis Angel ${ }^{1,2}$, Jun-Chieh J. Tsay ${ }^{1,2,4}$, Benjamin G. $W_{u^{1,2,4}}$, Stephen T. Yeung ${ }^{5}$, Kelsey Krolikowski ${ }^{1,2}$, Yonghua $L i^{1,2}$, Ralf Duerr ${ }^{5}$, Rosemary Schluger ${ }^{1,2}$, Sara A. Thannickal ${ }^{5}$, Akiko Koide ${ }^{2,6}$, Samaan Rafeq ${ }^{1,2}$, Clea Barnett $^{1,2}$, Radu Postelnicu ${ }^{1,2}$, Chang Wang ${ }^{7}$, Stephanie Banakis ${ }^{3}$, Lizzette PerezPerez $^{8}$, George Jour ${ }^{9}$, Guomiao Shen ${ }^{9}$, Peter Meyn ${ }^{10}$, Joseph Carpenito ${ }^{1,2}$, Xiuxiu Liu $^{1,10}$, Kun $\mathrm{Ji}^{1,11}$, Destiny Collazo ${ }^{1,2}$, Anthony Labarbiera, ${ }^{1,2}$, Nancy Amoroso ${ }^{1,2}$, Shari Brosnahan $^{1,2}$, Vikramjit Mukherjee ${ }^{1,2}$, David Kaufman, ${ }^{1,2}$, Jan Bakker ${ }^{1,2}$, Anthony Lubinsky, ${ }^{1,2}$, Deepak Pradhan ${ }^{1,2}$, Daniel H. Sterman ${ }^{1,2}$, Michael Weiden ${ }^{1,2}$, Adriana Hegu $^{9,12}$, Laura Evans ${ }^{13}$, Timothy M. Uyeki ${ }^{14}$, Jose C. Clemente ${ }^{15}$, Emmie De wit ${ }^{8}$, Ann Marie Schmidt ${ }^{16}$, Bo Shopsin ${ }^{17}$, Ludovic Desvignes ${ }^{5}$, Chan Wang ${ }^{18}$, Huilin $\mathrm{Li}^{18}$, Bin Zhang $^{19}$, Christian V. Forst ${ }^{19}$, Shohei Koide ${ }^{6,20}$, Kenneth A. Stapleford ${ }^{5}$, Kamal M. Khanna ${ }^{5,6}$, Elodie Ghedin ${ }^{3,7^{*}}$, and Leopoldo N. Segal ${ }^{1,2,6^{*}}$

${ }^{1}$ Division of Pulmonary and Critical Care Medicine, New York University Grossman School of Medicine, NYU Langone Health, New York, NY

${ }^{2}$ Department of Medicine, New York University Grossman School of Medicine, NYU Langone Health, New York, NY

${ }^{3}$ Systems Genomics Section, Laboratory of Parasitic Diseases, National Institute of Allergy and Infectious Diseases, National Institutes of Health, Bethesda, MD

${ }^{4}$ Division of Pulmonary and Critical Care Medicine, VA New York Harbor Healthcare System, NY

${ }^{5}$ Department of Microbiology, New York University Grossman School of Medicine, NYU Langone Health, New York, NY.

${ }^{6}$ Laura and Isaac Perlmutter Cancer Center, New York University School of Medicine, NYU Langone Health, New York, NY.

${ }^{7}$ Center for Genomics \& Systems Biology, Department of Biology, New York University New York, NY

${ }^{8}$ Molecular Pathology Unit, Laboratory of Virology, National Institute of Allergy and Infectious Diseases, National Institutes of Health, Rocky Mountain Laboratories, Hamilton, MT

${ }^{9}$ Department of Pathology, New York University Grossman School of Medicine, NYU Langone Health, New York, NY.

${ }^{10}$ Division of Pediatrics, Longhua Hospital Affiliated to Shanghai University of Chinese Medicine, CN

${ }^{11}$ Dongfang Hospital affiliated to Beijing University of Traditional Chinese Medicine, Beijing, China

${ }^{12}$ NYU Langone Genome Technology Center, New York University Grossman School of Medicine, NYU Langone Health, New York, NY

${ }^{13}$ Pulmonary, Critical Care and Sleep Medicine, University of Washington, Seattle, USA ${ }^{14}$ Influenza Division, Centers for Disease Control and Prevention, Atlanta, GA

${ }^{15}$ Department of Genetics and Genomic Sciences and Immunology Institute, Icahn School of Medicine at Mount Sinai, NY. 
16 Diabetes Research Program, Division of Endocrinology, Diabetes and Metabolism, Department of Medicine, New York University Grossman School of Medicine, NYU Langone Health, New York, NY

17 Division of Infectious Diseases, Department of Medicine, New York University School of Medicine, NYU Langone Health, New York, NY

18 Department of Population Health, New York University School of Medicine, NYU Langone Health, New York, NY

19 Department of Genetics and Genomic Sciences, Icahn Institute of Genomics and Multiscale Biology, Icahn School of Medicine at Mount Sinai, New York, NY

20 Department of Biochemistry and Molecular Pharmacology, New York University Grossman School of Medicine, NYU Langone Health, New York, NY and Perlmutter Cancer Center, New York, NY 10016

Imran Sulaiman, MD, $\mathrm{PhD}$ Matthew Chung, PhD

63 Luis Angel, MD

Jun-Chieh J. Tsay, MD

Benjamin G. Wu, MD Stephen Yeung, PhD Kelsey Krolikowski Yonghua Li, MD, PhD Ralf Duerr, MD, PhD Rosemary Schluger, RN Sara A. Thannickal, BS Akiko Koide, Phd, Samaan Rafeq, MD Clea Barnett, MD Radu Postelnicu, MD Chang Wang, PhD Stephanie Banakis Lizzette Perez-Perez George Jour, PhD Guomiao Shen, Phd Peter Meyn Joseph Carpenito Xiuxiu Liu, MS Kun Ji, MD, PhD Destiny Collazo Anthony Labarbiera Nancy Amoroso, MD Shari Brosnahan, MD Vikramjit Mukherjee, MD David Kaufman, MD Jan Bakker, MD, PhD Anthony Lubinsky, MD Deepak Pradhan, MD Daniel H. Sterman, MD
SheikMohammadlmran.Sulaiman@nyumc.org matt.chung@nih.gov

Luis.Angel@nyulangone.org

jun-chieh.tsay@nyumc.org

Benjamin.Wu@nyumc.org

Stephen.Yeung@nyulangone.org

Kelsey.Krolikowski@nyulangone.org

Yonghua.Li@nyumc.org

Ralf.Duerr@nyulangone.org rosemary.schluger@nyumc.org

Sara.thannickal@nyulangone.org

Akiko.Koide@nyulangone.org Samaan.Rafeq@nyulangone.org Clea.Barnett@nyulangone.org Radu.Postelnicu@nyulangone.org cw2505@nyu.edu stephanie.banakis@nih.gov lizzette.perez-perez@nih.gov George.Jour@nyulangone.org Guomiao.Shen@nyulangone.org peter.meyn@nyumc.org Joseph.carpenito@nyumc.org liuxiuxiu_longhua@outlook.com Kun.Ji@nyulangone.org Destiny.Collazo@nyulangone.org Anthony.Labarbiera@nyulangone.org Nancy.Amoroso@nyulangone.org Shari.Brosnahan@nyulangone.org Vikramjit.Mukherjee@nyulangone.org David.A.Kaufman@nyulangone.org Jan.Bakker@nyulangone.org Anthony.Lubinsky@nyulangone.org Deepak.Pradhan@nyulangone.org Daniel.sterman@nyumc.org 


$\begin{aligned} 95 & \text { Michael Weiden, MD } \\ 96 & \text { Adriana Heguy, PhD } \\ 97 & \text { Laura Evans, MD } \\ 98 & \text { Timothy M. Uyeki, MD, MPH } \\ 99 & \text { Jose C. Clemente, PhD } \\ 100 & \text { Emmie De wit } \\ 101 & \text { Ann Marie Schmidt, MD } \\ 102 & \text { Bo Shopsin, MD, PhD } \\ 103 & \text { Ludovic Desvignes, PhD } \\ 104 & \text { Chan Wang, PhD } \\ 105 & \text { Huilin Li, PhD } \\ 106 & \text { Bin Zhang, PhD } \\ 107 & \text { Christian V. Forst, PhD } \\ 108 & \text { Shohei Koide, PhD } \\ 109 & \text { Kenneth A. Stapleford, PhD } \\ 110 & \text { Kamal Khanna, PhD } \\ 111 & \text { Elodie Ghedin, PhD } \\ 112 & \text { Leopoldo N. Segal, MD } \\ 113 & \end{aligned}$

\author{
Michael.Weiden@nyulangone.org \\ Adriana.heguy@nyumc.org \\ leevans@uw.edu \\ tmu0@cdc.gov \\ jose.clemente@mssm.edu \\ emmie.dewit@nih.gov \\ AnnMarie.Schmidt@nyulangone.org \\ Bo.Shopsin@nyulangone.org \\ Ludovic.Desvignes@nyulangone.org \\ Chan.Wang@nyulangone.org \\ Huilin.Li@nyulangone.org \\ bin.zhang@mssm.edu \\ christian.forst@mssm.edu \\ Shohei.Koide@nyulangone.org \\ Kenneth.Stapleford@nyulangone.org \\ Kamal.Khanna@nyulangone.org \\ elodie.ghedin@nih.gov \\ Leopoldo.Segal@nyumc.org
}

113

${ }^{*}$ Corresponding Authors: Elodie Ghedin (elodie.ghedin@nih.gov) and Leopoldo N. Segal, (Leopoldo.Segal@nyumc.org)

\section{Authors Contributions:}

Study conception and design: IS, LA, LD, SK, KS, KK, EG, LNS

Data was obtained by: IS, LA, SY, KK, YL, RD, RS, SAT, AK, SR, CB, RP, GJ, GS, PM, $\mathrm{JC}, \mathrm{XL}, \mathrm{KJ}, \mathrm{DC}, \mathrm{AL}, \mathrm{NA}, \mathrm{SB}, \mathrm{VM}, \mathrm{DK}, \mathrm{JB}, \mathrm{AL}, \mathrm{DP}, \mathrm{LPP}, \mathrm{EDW}, \mathrm{DHS}, \mathrm{AH}, \mathrm{LNS}$

Data were analyzed by: IS, MC, JCJT, BGW, SY, KK, RD, CW, JCC, CW, HL, CVF, BZ, SK, KS, EG, LNS

The first draft of the manuscript was written by: IS, MC, LA, EG, LNS

All authors read, critically revised and approved the final manuscript.

\section{Research support funding:}

R37 CA244775 (LNS, NCI/NIH); R01 HL125816 (LNS, SBK, NHLBI/NIH); Colton Pilot Project Grant (LNS, LA, SBL, KK); UWSC12030 (LNS, RP, LE, CDC Foundation); PACT grant (LNS, FNIH); R21 Al158997 (SK); R01 Al143861 (KMK, NIAID/NIH); R01 Al143861-02S1 (KMK, NIAID/NIH); R01 DK110014 (HL and CW, NIDDK/NIH); P20 CA252728 (CW and HL, NCl/NIH) American Association for Cancer Research Grant (HP/LNS); The Genome Technology Center is partially supported by the Cancer Center Support Grant P30CA016087 at the Laura and Isaac Perlmutter Cancer Center (AH, AT); FAMRI Young Clinical Scientist Award (BGW), Stony Wold-Herbert Fund Grant-inAid/Fellowship (IS, CB). This work was supported in part by the Division of Intramural Research (DIR) of the NIAID/NIH (EG, EDW).

\section{Acknowledgement:}

We would like to thank the Genome Technology Center (GTC) for expert library preparation and sequencing, and the Applied Bioinformatics Laboratories (ABL) for providing bioinformatics support and helping with the analysis and interpretation of the 
143 data. Experimental Pathology Research Laboratory for histopathology services and 144 imaging. GTC and ABL are shared resources partially supported by the Cancer Center 145 Support Grant P30CA016087 at the Laura and Isaac Perlmutter Cancer Center. This 146 work has used computing resources at the NYU School of Medicine High Performance 147 Computing Facility (HPCF). Financial support for the PACT project is possible through 148 funding support provided to the FNIH by: AbbVie Inc., Amgen Inc., Boehringer149 Ingelheim Pharma GmbH \& Co. KG, Bristol-Myers Squibb, Celgene Corporation, 150 Genentech Inc., Gilead, GlaxoSmithKline plc, Janssen Pharmaceutical Companies of 151 Johnson \& Johnson, Novartis Institutes for Biomedical Research, Pfizer Inc., and 152 Sanofi. The findings and conclusions in this report are those of the authors and do not 153 necessarily represent the official position of the Centers for Disease Control and 154 Prevention.

158 Running Head: COVID-19

159

Word Count: 4869

162 Body of the manuscript:

Financial Disclosure: None

Key words: microbiome, virome, severe acute respiratory syndrome 
medRxiv preprint doi: https://doi.org/10.1101/2021.02.23.21252221; this version posted February 26, 2021. The copyright holder for this preprint (which was not certified by peer review) is the author/funder, who has granted medRxiv a license to display the preprint in perpetuity.

All rights reserved. No reuse allowed without permission.

\section{Abstract:}

176 Mortality among patients with COVID-19 and respiratory failure is high and there are no known

177 lower airway biomarkers that predict clinical outcome. We investigated whether bacterial

178 respiratory infections and viral load were associated with poor clinical outcome and host

179 immune tone. We obtained bacterial and fungal culture data from 589 critically ill subjects with

180 COVID-19 requiring mechanical ventilation. On a subset of the subjects that underwent

181 bronchoscopy, we also quantified SARS-CoV-2 viral load, analyzed the microbiome of the lower

182 airways by metagenome and metatranscriptome analyses and profiled the host immune

183 response. We found that isolation of a hospital-acquired respiratory pathogen was not

184 associated with fatal outcome. However, poor clinical outcome was associated with enrichment

185 of the lower airway microbiota with an oral commensal (Mycoplasma salivarium), while high

186 SARS-CoV-2 viral burden, poor anti-SARS-CoV-2 antibody response, together with a unique

187 host transcriptome profile of the lower airways were most predictive of mortality. Collectively,

188 these data support the hypothesis that 1) the extent of viral infectivity drives mortality in severe

189 COVID-19, and therefore 2) clinical management strategies targeting viral replication and host

190 responses to SARS-CoV-2 should be prioritized.

191

192

193

194

195

196

197 


\section{Introduction}

199 The earliest known case of Severe Acute Respiratory Syndrome Coronavirus 2 (SARS-

200 CoV-2) infection causing coronavirus virus disease (COVID-19) is thought to have 201 occurred on November 17, 2019¹. As of February 20, 2021, 110.3 million confirmed

202 cases of COVID-19 and 2.4 million deaths have been reported worldwide ${ }^{2}$. As the

203 global scientific community rallied in a concerted effort to understand SARS-CoV-2

204 infections, our background knowledge is rooted in previous experience with the related

205 zoonotic betacoronaviruses Middle East Respiratory Syndrome coronavirus (MERS-

206 CoV) and SARS-CoV-1 that have caused severe pneumonia with $34.4 \%$ and $9 \%$ case

207 fatality, respectively ${ }^{3}$. As observed for these related coronaviruses, SARS-CoV-2

208 infection can result in an uncontrolled inflammatory response ${ }^{4}$ leading to acute

209 respiratory distress syndrome (ARDS) and multi-organ failure, both associated with

210 increased mortality. While a large proportion of the SARS-CoV-2 infected population is

211 asymptomatic or experiences mild illness, a substantial number of individuals will

212 develop severe disease and require hospitalization, with some progressing to

213 respiratory failure. Mortality among hospitalized COVID-19 patients is estimated to be

214 approximately $20 \%$, which can go up to $70 \%$ among those requiring invasive

215 mechanical ventilation ${ }^{5-12}$.

217 Mortality in other viral pandemics, such as the $1918 \mathrm{H} 1 \mathrm{~N} 1$ and $2009 \mathrm{H} 1 \mathrm{~N} 1$ influenza

218 pandemics, has been attributed in part to bacterial co-infection or super-infection ${ }^{13,14}$.

219 To determine if this is also the case for COVID-19, we can use next generation

220 sequencing (NGS) to probe the complexity of the microbial environment (including RNA

221 and DNA viruses, bacteria and fungi) and how the host (human) responds to infection. 
222 Recent studies have used this approach to uncover microbial signatures in patients with

223 ARDS. ${ }^{15,16}$ Increased bacterial burden and the presence of gut-associated bacteria in

224 the lung were shown to worsen outcomes in these critically ill patients ${ }^{15,17}$, highlighting

225 the potential role of the lung microbiome in predicting outcomes in ARDS. In a recent

226 study using whole genome sequencing to profile the gut microbiome of 69 patients from

227 Hong Kong, investigators identified an increased abundance of opportunistic fungal

228 pathogens among patients with confirmed COVID-19 ${ }^{18}$. While there is emerging interest

229 in understanding the microbial environment in patients with SARS-CoV-2 infections, few

230 studies have attempted to characterize this at the primary site of the disease activity:

231 the lower airways ${ }^{19,20}$. Furthermore, no study has yet determined whether microbial

232 differences in the airways of COVID-19 patients could be contributing to the different

233 outcomes in patients receiving mechanical ventilation.

235 In this investigation, we accessed a large prospective cohort of critically ill patients with

236 SARS-CoV-2 infection who required invasive mechanical ventilation, and from whom

237 bronchoalveolar lavage $(\mathrm{BAL})$ samples were collected. We characterized the lung

238 microbiome of these patients in parallel with analyses of lower airway markers of host

239 immunity. While we did not find that isolation of a secondary respiratory pathogen was

240 associated with prolonged mechanical ventilation (>28 days) or fatal outcome, we did

241 identify critical microbial signatures-characterized by enrichment of oral commensals,

242 high SARS-CoV-2 load, and decreased anti-SARS-CoV-2 IgG response-associated

243 with fatal outcome, suggesting a need for more targeted antiviral therapeutic

244 approaches for the care of critically ill COVID19 patients. 


\section{Results}

247 Cohort

248 From March $3^{\text {rd }}$ to June $18^{\text {th }} 2020$, a total of 589 patients with laboratory-confirmed 249 SARS-CoV-2 infection were admitted to the intensive care units of two academic

250 medical centers of NYU Langone Health in New York (Long Island and Manhattan) and

251 required invasive mechanical ventilation (MV). This included a subset of 142 patients

252 from the Manhattan campus who underwent bronchoscopy for airway clearance and/or

253 tracheostomy from which we collected and processed lower airway samples for this

254 investigation (Supplementary Fig. 1). Table 1 shows demographics and clinical

255 characteristics of the 142 patients who underwent bronchoscopy divided into three

256 clinical outcomes: survivors with $\leq 28$ Days on MV; survivors with $>28$ Days on MV; and

257 deceased. The median post admission follow-up time was 232 days ( $\mathrm{Cl}=226-237$ days).

258 Supplementary Tables 1 and 2 compare similar data across all 589 subjects, divided

259 per site and sub-cohorts. Patients at the Manhattan campus who underwent

260 bronchoscopy were younger, had lower body mass index (BMI), and a lower prevalence

261 of chronic obstructive pulmonary disease (COPD; Supplementary Table 1). Among the

262 cohort that provided lower airway samples through bronchoscopy, $37 \%$ of the subjects

263 were successfully weaned within 28 days of initiation of MV and survived

264 hospitalization, 39\% required prolonged MV but survived hospitalization, and 23\% died.

265 Patients within the bronchoscopy cohort had a higher overall survival than the rest of

266 the NYU COVID-19 cohort since most critically ill patients were not eligible for

267 bronchoscopy or tracheostomy. Mortality among those in the no-bronchoscopy cohort

268 was 77\%. In the overall NYU cohort, higher age and BMI were associated with

269 increased mortality (Supplementary Table 2). There was a similar, albeit non- 
270 significant, trend for the bronchoscopy cohort. Among the clinical characteristics of this

271 cohort, patients within the deceased group more commonly had a past medical history

272 of chronic kidney disease and cerebrovascular accident.

273 Study patients were admitted during the first wave of the pandemic in the US, prior to

274 current standardized management of COVID-19. Within the bronchoscopy cohort, more

275 than $90 \%$ of the subjects received hydroxychloroquine and anticoagulation (therapeutic

276 dose), 69\% received corticosteroids, 41\% received tocilizumab (anti-Interleukin (IL)-6

277 receptor monoclonal antibody), $21 \%$ required dialysis, and $18.9 \%$ were started on

278 extracorporeal membrane oxygenation (ECMO) (Table 1). Antimicrobial therapy

279 included use of antivirals (lopinavir/ritonavir in 16\% and remdesivir in 10\%), antifungals

280 (fluconazole in $40 \%$ and micafungin in 57\%), and antibiotics (any, in $90 \%$ of the

281 subjects). Among the factors associated with clinical outcome within the bronchoscopy

282 cohort, patients who survived were more commonly placed on ECMO whereas patients

283 who died had frequently required dialysis (Table 1); these trends were also observed

284 across the whole NYU cohort. Neither hydroxychloroquine or azithromycin were

285 significantly associated with clinical outcome; however, patients who survived were

286 more frequently treated with the combination antibiotic piperacillin/tazobactam.

288 Within the first $48 \mathrm{hrs}$ from admission, respiratory bacterial cultures were rarely obtained $289(n=70 / 589,12 \%)$ with very few positive results $(n=12,17 \%)$. Blood cultures were more 290 commonly obtained $(n=353 / 589,60 \%)$ but the rate of bacterial culture positivity was

291 much lower $(n=5,1.4 \%)$. These data support that community acquired bacterial co-

292 infection was not a common presentation among critically ill COVID-19 patients. 
294 During their hospitalization, most patients had respiratory and/or blood specimens

295 collected for bacterial cultures (Table 1 and Supplementary Table 1). The proportions

296 of positive bacterial respiratory cultures and blood cultures were $73 \%$ and $43 \%$,

297 respectively. We evaluated whether respiratory or blood culture results obtained as per

298 clinical standard of care were associated with clinical outcome. Risk analyses for the

299 culture results during hospitalization for the whole cohort $(n=589)$ demonstrated that

300 bacterial culture positivity was not associated with increased odds of dying but was

301 associated with prolonged mechanical ventilation in the surviving patients (Figure 1).

302 Since length of stay could potentially affect these results (patients who died could have

303 a shorter hospitalization, and therefore may have had fewer specimens collected for

304 cultures), we repeated the analysis using culture data obtained during the first two

305 weeks of hospitalization. This analysis showed that bacterial pathogen culture positivity

306 (both respiratory and blood) during the early period of hospitalization was not

307 associated with worse outcome (Figure 1 and Supplementary Table 3). Interestingly,

308 identification of oral bacteria in respiratory culture, commonly regarded as procedural

309 contaminants, was associated with higher odds of prolonged mechanical ventilation

310 (>28 days) among survivors. Similar trends were noted when analysis was performed

311 on subjects from NYU LI and NYU Manhattan separately, or for the bronchoscopy

312 cohort (Supplementary Table 2). Among the bronchoscopy cohort, there was no

313 statistically significant association between culture results and clinical outcome, but

314 there was a trend towards an increased rate of positive respiratory cultures for

315 Staphylococcus aureus (including MRSA), S. epidermidis, and Klebsiella pneumoniae in

316 the survival groups (Table 1). These data suggest that in critically ill patients with

317 COVID-19 requiring MV, hospital isolation of a secondary respiratory bacterial pathogen 
318 is not associated with worse clinical outcome.

320 SARS-CoV-2 load in the lower airways is associated with poor clinical outcome

321 Using supraglottic and BAL samples from patients undergoing bronchoscopy $(n=142)$,

322 we evaluated the viral load by rRT-PCR for the SARS-CoV-2 N gene, adjusted by levels

323 of human ribosomal protein (RP). Of note, the majority of samples were largely obtained

324 in the second week of hospitalization (Table 1, median[IQR] = 10[6-14], 13[8-16], and

325 13[8-16] for the $\leq 28$-days MV, >28-days MV, and deceased groups, respectively, $\mathrm{p}=\mathrm{ns}$ ).

326 Paired analysis of upper and lower airway samples revealed that, while there was a

327 positive association between SARS-CoV-2 viral load of the paired samples (rho $=0.60$,

$328 \mathrm{p}<0.0001)$, there was a subset of subjects $(21 \%)$ for which the viral load was greater in

329 the BAL than in the supraglottic area, indicating topographical differences in SARS-

330 CoV-2 replication (Figure 2a). Importantly, while the SARS-CoV-2 viral load in the

331 upper airway samples was not associated with clinical outcome (Supplementary Fig.

332 2), patients who died had higher viral load in their lower airways than patients who

333 survived (Figure 2b). We then evaluated virus viability in BAL samples by measuring

334 levels of subgenomic RNA (sgRNA) targeting the E gene of SARS-CoV-2. This mRNA

335 is only transcribed inside infected mammalian cells and is not packed into virions, thus,

336 its presence is indicative of viable infecting viral particles in a sample ${ }^{21}$. In BAL, levels of

337 sgRNA correlated with viral load as estimated by rRT-PCR for the SARS-CoV-2 N gene

338 (Figure 2c) and the highest percentage of measurable sgRNA was in the deceased

339 group followed by the $\leq 28$-days MV group, and the $>28$-days MV group (17,7\%, 11.5\%,

340 and $3.7 \%$, respectively, chi-square $p=0.028$ for the comparison deceased vs. $>28$-days

341 MV group). Thus, while in most cases levels of sgRNA were not measurable in BAL 
342 suggesting that there were no viable viral particles in the lower airways of COVID-19

343 patients at the time of bronchoscopy (overall median[IQR] = 12[7-16] days from

344 hospitalization), the lower airway viral burden, as estimated by rRT-PCR, is associated

345 with mortality in critically ill COVID-19 patients.

347 Microbial community structure of the lower airways is distinct from the upper airways in

348 critically ill patients.

349 Considering the bacterial species and the viral loads identified in the lower and upper

350 airways of this cohort and their association with outcomes, we profiled in detail their viral

351 and microbial composition. Microbial communities were evaluated using parallel

352 datasets of RNA and DNA sequencing from 118 COVID-19 patients with lower airway

353 samples that passed appropriate quality control and a subset of paired 64 upper airway

354 samples, along with background bronchoscope controls.

356 RNA sequencing (RNAseq) of the metatranscriptome provided insight into the RNA

357 virome as well as the transcriptomes of DNA viruses, bacteria, and fungi. Given the low

358 biomass of lower airway samples we first identified taxa as probable contaminants by

359 comparing the relative abundance between background bronchoscope and BAL

360 samples (Supplementary Fig. 3a and Supplementary Table 4). However, we did not

361 remove any taxa identified as probable contaminants from subsequent analyses. A

362 comparison of the microbial community complexity captured in these data, determined

363 using the Shannon diversity Index, showed there was significantly lower $\alpha$ diversity in

364 the lower airway samples than in the upper airways and background controls

365 (Supplementary Fig. 4a). Similarly, $\beta$ diversity analysis based on the Bray Curtis 
366 Dissimilarity index indicated that the microbial composition of the lower airways was

367 distinct from the upper airways and background controls (Supplementary Fig. 4b,

368 PERMANOVA $p<0.01$ ). Sequence reads indicated a much higher relative abundance of

369 SARS-CoV-2 in the lower than in the upper airways for this cohort (Supplementary Fig.

370 4c). Comparisons of the most dominant bacterial and fungal taxa that were functionally

371 active showed that $S$. epidermidis, Mycoplasma salivarium, S. aureus, Prevotella oris,

372 and Candida albicans, many often-considered oral commensals, were present in both

373 upper and lower airway samples (Supplementary Fig. 4c). Interestingly, the lytic phage

374 Proteus virus Isfahan, known to be active against biofilms of Proteus mirabilis ${ }^{22}$, was

375 found to be highly transcriptionally active in the BAL.

377 DNA sequencing data provided insight into the DNA virome, as well as the bacterial and

378 fungal metagenomes. As for the metatranscriptome data, we first identified taxa as

379 probable contaminants but these were not removed for subsequent analyses

380 (Supplementary Fig. 3b). Both $\alpha$ and $\beta$ diversity analyses of the metagenome support

381 distinct microbial community features in the lower airways as compared with the upper

382 airways and background controls (Supplementary Fig. 5a, 5b). Among the top 10 taxa

383 across lower and upper airway samples were S. aureus, Salmonella enterica,

384 Burkholderia dolosa, and Klebsiella variicola. Candida albicans only ranked \#77 in the

385 BAL while it was ranked $5^{\text {th }}$ in the metatranscriptome data indicating that while present

386 at low relative abundance, it was highly active (Supplementary Table 4). K. variicola,

387 while prevalent at a high relative abundance (\#4 in BAL, and \#5 in the upper airways) in

388 patients of this cohort, its ranking in the RNAseq data was not among the top 50,

389 indicating that it was not as active functionally as other bacteria. Conversely, while $S$. 
390 epidermis ranked as the most highly functional taxon in both lower and upper airways,

391 based on RNAseq reads (Supplemental Fig. 3c), it was $33^{\text {rd }}$ in relative abundance in

392 the BAL DNAseq data but was present at very high relative abundance in the upper

393 airways (ranked \#3). These data suggest that microbes that colonize the upper airways

394 and the skin were common in the lower airways in this cohort of COVID-19 patients

395 requiring invasive mechanical ventilation.

397 Distinct microbial signatures are associated with different clinical outcomes.

398 To determine the potential impact of vertebrate viruses on outcome, we compared virus

399 enrichment differences in BAL samples across the three clinical outcome groups $(\leq 28$ -

400 days $\mathrm{MV},>28$-days $\mathrm{MV}$, and deceased). As it pertains to the vertebrate RNA virome

401 subfraction, there were significant differences ( $\beta$ diversity) between the three clinical

402 outcome groups (Supplementary Fig. 6, PERMANOVA $p<0.01$ ). There were no

403 significant differences for the vertebrate DNA virome or DNA virus transcriptome

404 subfractions of the sequence reads (data not shown). Consistent with the SARS-CoV-2

405 viral load assessed by RT-PCR, differential expression analysis (DESeq) of the RNA

406 virome identified SARS-CoV-2 as being enriched in the deceased group, as compared

407 with both $\leq 28$-days and $>28$-days MV groups (fold change $>5$, Figure 2 d). Cox

408 proportional hazards modeling supports that enrichment with SARS-CoV-2 was

409 associated with increased risk for death $(\mathrm{HR} 1.33,95 \% \mathrm{Cl}=1.07-1.67$, pvalue=0.011,

410 FDR adjusted pvalue=0.06; Supplementary Table 5).

412 Analysis of differential DNA virus abundance using DEseq did not show statistically 413 significant differences. Because the virome includes viruses of bacteria and archaea, 
414 we also analyzed the phage data (including viruses of archaea). Phages impact the

415 bacterial population-including bacterial pathogens_and so could be clinically relevant.

416 At a compositional level, the virome of DNA phages did not display statistically

417 significant differences or significant virus enrichment based on clinical outcome groups

418 (data not shown). However, while the phage metatranscriptome $\alpha$ and $\beta$ diversity was

419 similar across the clinical outcome groups, there were various taxonomic differences at

420 the RNA level with enrichment of Staphylococcus phages CNPx in the deceased and

421 >28-day MV groups when compared with the $\leq 28$-day MV group (Figure 2e).

422 Differential expression from two other Staphylococcus phages was also observed in the

423 >28-days MV group as compared with the $\leq 28$-days MV group (Figure 2e). None of the

424 described taxa were identified as possible contaminants (Supplementary Table 4).

426 Enrichment of the lower airway microbiota with oral commensals is associated with poor

\section{7 outcome}

428 We evaluated the overall bacterial load by quantitative PCR, targeting the 16S rRNA

429 gene. As expected, the bacterial load in the lower airways was several folds lower than

430 in the upper airways but clearly higher than the background bronchoscope control

431 (Supplementary Fig. 7). Patients who died had higher total bacterial load in their lower

432 airways than patients who survived (Figure 3a).

434 While no statistically significant differences were noted in $\alpha$ or $\beta$ diversity across clinical

435 outcome groups (Figure $3 \mathbf{b}-\mathbf{c}$ ), several differences were noted when differential

436 enrichment was evaluated using DESeq. For the comparisons made across the clinical

437 outcome groups we focused on consistent signatures identified in the lower airway 
438 metagenome and metatranscriptome. Coherence of differentially enriched taxa was

439 determined by gene set enrichment analysis (GSEA) (Figure 3d) and directionality of

440 enrichment between the two datasets was evaluated (Figure 3e). Among the most

441 abundant taxa, the oral commensal $M$. salivarum was enriched in the deceased and

442 >28-days MV groups as compared with the $\leq 28$-days MV group. In contrast, a different

443 oral commensal, Prevotella oris, was enriched in the $\leq 28$-days MV group as compared

444 with the deceased and >28-days MV groups. These data support that oral commensals

445 are frequently found in the lower airways of critically ill COVID-19 patients and that

446 differences between groups could be due to differential microbial pressures related to

447 host factors. Interestingly, most of the statistically significant taxa were identified in the

448 metatranscriptome rather than in the metagenome data, with only $P$. oris identified in

449 both datasets. None of the described taxa were identified as possible contaminants

450 (Supplementary Table 4). Overall, most of the microbial signatures identified as

451 enriched in the deceased or in subjects on prolonged MV are regular colonizers of

452 healthy skin and mucosal surfaces rather than frequent respiratory pathogens.

454 For the fungal data, there were no statistically significant differences in $\alpha$ or $\beta$ diversity 455 identified between clinical outcome groups in the metagenome or the

456 metatranscriptome data (Supplementary Fig. 8a and 8c). However, in the 457 metagenome data, we identified Candida glabrata enriched in the deceased group as 458 compared with the $\leq 28$-days MV and the $>28$-days MV groups but this was not 459 consistent in the metatranscriptome data (Supplementary Fig. 8b and 8d). 
medRxiv preprint doi: https://doi.org/10.1101/2021.02.23.21252221; this version posted February 26, 2021. The copyright holder for this preprint (which was not certified by peer review) is the author/funder, who has granted medRxiv a license to display the preprint in perpetuity.

All rights reserved. No reuse allowed without permission.

461 Poor clinical outcomes are associated with enrichment of antimicrobial resistance genes

462 and glycosphingolipid biosynthesis

463 We used the gene annotation of the DNAseq and RNAseq data to profile the microbial

464 functional potential of the lower airway samples. For the comparisons made across the

465 clinical outcome groups, we focused on consistent functional signatures identified in the

466 lower airway metagenome and metatranscriptome. Coherence of differentially enriched

467 functions was determined using GSEA (Figure 4a) and directionality of enrichment was

468 also evaluated (Figure 4b). Overall, there was coherence of directionality between the

469 metranscriptomics and metagenomics datasets for the comparisons between deceased

470 vs $\leq 28$-days MV, and $>28$-days MV vs $\leq 28$-days MV groups. Interestingly, statistically

471 significant differences were only noted in the metatranscriptome data and not in the

472 metagenome data. Among the top differentially expressed pathways in the poor

473 outcome groups were glycosylases, oxidoreductase activity, transporters, and two-

474 component system, among other genes. The two-component system is used by

475 bacteria and fungi for signaling. A specific analysis of antibiotic resistance genes shows

476 that there was significant gene enrichment and expression of biocide resistance in the

477 deceased group as compared to the two other MV groups (Supplementary Fig. 9).

478 There was also significant expression of genes resistant to trimethoprim and phenolic

479 compound, as well as multi drug resistance in the deceased group as compared to the

$480 \leq 28$-days MV group. Presence of the resistance gene against Trimethropim was not

481 significantly associated with prior exposure with Trimethoprim. However, only 7 patients

482 received this drug before sample collection. 
medRxiv preprint doi: https://doi.org/10.1101/2021.02.23.21252221; this version posted February 26, 2021. The copyright holder for this preprint (which was not certified by peer review) is the author/funder, who has granted medRxiv a license to display the preprint in perpetuity.

All rights reserved. No reuse allowed without permission.

484 Lower airway host immune phenotype shows failure of adaptive and innate immune

485 response to SARS-CoV-2 among deceased subjects

486 To evaluate the host immune response to SARS-CoV-2 infection, we first measured

487 levels of anti-Spike and anti-RBD (receptor binding domain) antibodies in BAL samples.

488 For both anti-Spike and anti-RBD immunoglobulins, levels of $\lg$, $\lg A$ and $\lg M$ were

489 several logs higher than levels found in BAL samples from non-SARS-CoV-2 infected

490 patients. Importantly, IgG levels of anti-Spike and anti-RBD were significantly lower in

491 the deceased group as compared to the levels found in patients who survived (Figure

492 5a and Supplementary Fig. 10a-c, p<0.05). A neutralization assay performed using

493 BAL fluid showed varying levels of neutralization across all samples (as estimated by

494 EC50) but no statistically significant differences between the clinical outcome groups

495 (Supplementary Fig. 10d). We then evaluated whether levels of antibodies correlated

496 with viral load in BAL samples. While viral load levels of SARS-CoV-2 measured with

497 rRT-PCR did not correlate with BAL measurements of SARS-CoV-2 specific antibodies,

498 sgRNA viral load levels negatively correlated with BAL levels of Anti-Spike (IgG and

$499 \lg A)$, Anti-RBD (IgG and $\lg A)$ and the Neutralization assay (Supplementary Table 6).

500 These data suggest that the IgG subfraction is an important marker of the adaptive

501 immune response in the lung of critically ill COVID-19 patients and that both sub-

502 fractions of $\operatorname{lgG}$ and $\lg A$ anti-SARS-CoV-2 may contribute to the viral replication control

503 in the lower airways.

504

505 Host transcriptome analyses of BAL samples showed significant differences across 506 clinical outcome groups based on $\beta$ diversity composition (Supplementary Fig. 11). We

507 identified multiple differentially expressed genes across the clinical outcome groups 
508 (Supplementary Fig. 11b-d). First, we noted that the lower airway transcriptomes

509 showed downregulation of heavy constant of $\lg$ (IGHG3), and heavy constant of $\lg A$

510 (IGHA1) genes in those with worse clinical outcome (Supplementary Table 7). We

511 then used IPA (Ingenuity Pathway Analysis) to summarize differentially expressed

512 genes across the three clinical outcome groups (Figure 5b). The sirtuin Signaling

513 Pathway (a pathway known to be involved in aging, gluconeogenesis/lipogenesis, and

514 host defense against viruses) ${ }^{23}$ and the ferroptosis pathway (an iron-dependent form of

515 regulated cell death present in bronchial epithelium) ${ }^{24,25}$ were both upregulated in those

516 with worse outcome. While this may reflect the host response to viral infection, other

517 differences in the transcriptomic data showed downregulation of mitochondrial oxidative

518 phosphorylation, HIF1a, STAT3, and Phospholipase C Signaling. Additional canonical

519 signaling pathways, including insulin secretion, multiple Inositol related pathways,

520 noradrenaline/adrenaline degradation signaling, and xenobiotic related metabolism

521 were significantly downregulated when comparing the $>28$-days MV vs. $\leq 28$-days MV

522 groups. Upstream pathway prediction analyses of the host airway transcriptome support

523 previously reported mitochondria dysfunction ${ }^{26}$ (inhibition in mitochondrial related

524 regulators NSUN3, MRPL14, MRPL12, LONP1, DAP3), and metabolic/gluconeogenesis

525 dysregulation ${ }^{27,28}$ (SIRT3) in critically ill COVID-19 subjects with poor outcome

526 (Supplementary Table 8). We also observed decreased activation in the inflammatory

527 response in critically ill COVID-19 subjects with poor outcome (phagocytes, neutrophils,

528 and granulocytes, and leukocytes; Supplementary Table 9). A comparison of clinical

529 outcome between the >28-days MV vs. $\leq 28$-days MV groups showed upstream

530 predicted inhibition in insulin, estrogen, beta-estradiol, EGF, EGFR, IL-5, and IL-10RA

531 in the >28-days MV group (Supplementary Table 9). These differences suggest that, at 
532 the stage that we sampled the lower airways of patients with critically COVID-19, an

533 overt inflammatory tone was not predictive of worst outcome.

535 To determine if the abundance of immune cells varies between different clinical

536 outcome groups, we estimated cell type abundance from the host transcriptome with

537 computational cell type quantification methods, including a deconvolution approach

538 implemented in CIBERSORTx ${ }^{29}$ and a cell type signature enrichment approach

539 implemented in $x$ Cell ${ }^{30}$. As reported recently in other studies ${ }^{31}$, among the cell types

540 detected in the BAL samples we observed a consistent enrichment of mast cells and

541 neutrophils in the $>28$-days MV and deceased groups compared with the $\leq 28$-days MV

542 group (Figure 5c and Supplementary Table 10). We also identified significantly higher

543 inflammatory macrophages (M1), innate T-cells and memory T-cells $\left(\mathrm{CCR} 7^{+}\right)$among

544 subjects with worse clinical outcome.

546 Cross-kingdom network analyses identify bacteria, fungi, and host pathways functionally

547 impacted by SARS-CoV-2

548 To identify potential microbe-microbe and microbe-host interactions that could have an

549 effect on outcome, we used a multi-scale network analysis approach (Multiscale

550 Embedded Gene co-Expression Network Analysis, MEGENA) ${ }^{32}$. We first used the

551 relative abundance from the RNAseq data to capture co-expressing taxa in the

552 metatranscriptome network neighborhood of SARS-CoV-2 (SARS2-NWN). We

553 examined five such network neighborhoods (constructed by including nodes with

554 increasing distance 1 to 5 from SARS-CoV-2, i.e. neighborhood 1 to neighborhood 5)

555 that were significantly enriched for taxa functionally active in the deceased group when 
556 compared with the $\leq 28$-day MV group. Only the largest cluster, with 504 taxa, had

557 significantly enriched taxa in both the deceased and in the $\leq 28$-day MV outcome groups

558 (Supplementary Fig. 12a) (FET P-value $=4.6 \mathrm{e}-45,4.0 \mathrm{FE})$. Many of these taxa are

559 among the top 50 most abundant microbes we had previously identified in the

560 metatranscriptome dataset. Taxa present that are influenced by SARS-CoV-2 and

561 significantly differentially enriched in the deceased group include bacteria such as $M$.

562 salivarium, Bifidobacterium breve, and Lactobacillus rhamnosus (a gut commensal),

563 that we had previously identified by differential expression analysis (Figure 3e), but also

564 taxa such as S. epidermis, Mycoplasma hominis (urogenital bacteria), and the phage

565 VB_PmiS-Isfahan (also referred to as Proteus virus Isfahan) that we had previously

566 only picked up as being highly abundant but not necessarily differentially enriched in the

567 deceased group. Most of the fungi, such as C. albicans, C. glabrata and C. orthopsilosis

568 were enriched in the $\leq 28$-day MV group. Interestingly, our earlier analysis of the

569 metagenome (Supplementary Fig. 8b) had identified C. glabrata as being enriched in

570 the deceased group with no enrichment in the metatranscriptome. This analysis

571 indicates that some of these abundant taxa could be responding to SARS-CoV-2

572 disruption in a similar manner, or indirectly interacting functionally.

573

574 We further investigated the association of the network neighborhood with host network

575 modules using the host transcriptome data to identify groups of host genes that are co-

576 expressed in response to SARS-CoV-2 disruption. The 3 host modules with the most

577 significant correlations to SARS2-NWN are M175, M277 and M718. M277 is the parent

578 module of $\mathrm{M718}$, and both are enriched with genes related to respiratory electron

579 transport, while M175 is enriched for IFN-Y signaling (Supplementary Fig. 12b). 
580 Module M175 is positively correlated with the SARS2-NWN $(\rho=0.32, P$-value $=2.1 e-3)$.

581 While there was no collective enrichment of the module by differentially expressed

582 genes (DEGs) in the deceased vs $\leq 28$-days MV, there was for $>28$-days vs $\leq 28$-days

583 MV (FET P-value $=0.030,4.5 \mathrm{FE}$ ). This module includes well-known antiviral IFN

584 stimulated genes (ISGs), such as IRF7 and OASL. Investigating module response on an

585 individual gene level, Interleukin 4 induced 1 (IL4I1) appears as one of the most up-

586 regulated genes in this module when comparing the deceased group with the $\leq 28$-day

587 MV group. The transporter 1, ATP binding cassette subfamily B member (TAP1) is also

588 upregulated and a key regulator (hub gene). Together with TAP2, TAP1 plays a central

589 role in $\mathrm{MHC}$ I antigen presentation ${ }^{33}$. Transcriptional regulators SP110 and SP140, both

590 ISGs and also identified as hub genes, were down-regulated. Module 718 was also

591 positively correlated with the SARS2-NWN ( $\rho=0.31$, P-value = 1.3e-3; enrichment FET

592 P-value $=0.029,3.7$ FE of M178 by differentially expressed genes in deceased vs $\leq 28-$

593 days MV). The majority of genes in this module are down-regulated in the deceased

594 group compared with the s28-day group. Some of the genes encode subunits of the

595 mitochondrial ATP synthase, such as ATP6 and ATP8, the cytochrome C oxidase, with

596 COX2 and COX3 as well as the NADH dehydrogenase complex, such as ND1-ND6.

597 ND4L, ATP6, COX2, ND1, ND3, ND4L and ND6 are key regulators, potentially

598 modulating the expression of the other genes in the module. These findings further

599 support mitochondria dysfunction ${ }^{26}$, potentially disrupting processes indicated by the

600 module. Other down-regulated genes are humanin1 (MTRNR2L1) and R-spondin 1

601 (RSPO1). Humanin is known to protect against oxidative stress and mitochondrial

602 dysfunction ${ }^{34}$. RSPO1 protects against cell stress by activating the Wnt/ $\beta$-catenin 603 signaling pathway ${ }^{35}$. Non-coding RNAs, such as MALAT1 and RHOQ-AS1 were found 
604 to be up-regulated. MALAT1 is known to suppress IRF3-initiated antiviral innate

605 immunity ${ }^{36}$ while the function of $R H O Q-A S 1$ is unknown.

608 Metatranscriptome and Transcriptome signatures are predictive of mortality

609 We evaluated the strength of the metatranscriptomic, metagenomic and host

610 transcriptomic profiles to predict mortality in this cohort of critically ill COVID-19 patients.

611 To this end, we identified features in each of these datasets and constructed risk scores

612 that best predicted mortality. Figure 6a shows that the metatranscriptome data, alone or

613 combined with the other two datasets, was most predictive of mortality. Importantly, the

614 predictive power (as estimated by the area under the curve) of the metatranscriptome

615 data was improved by excluding probable contaminants and worsened when SARS-

616 CoV-2 was removed from the modeling. The selected features we used to construct the

617 metatranscriptome, metagenome and host transcriptome risk scores are reported in

618 Supplementary Table 11). Using the means of the scores, we classified all subjects

619 into high risk and low risk groups for mortality. Figure 6b shows Kaplan-Meier survival

620 curve comparisons evaluating the predictive power of risk score stratification based on

621 metatranscriptome, metagenome and host transcriptome data. Combining risk scores

622 from different datasets showed an optimal identification of mortality when

623 metatranscriptome and host transcriptome were considered (Figure 6c). We then used

624 the gene signature found as being the most predictive of mortality to conduct IPA

625 analyses. Among the upstream regulators, mortality was associated with predicted

626 activation of interferon alpha while chemotaxis and infection by RNA virus were

627 predicted as activated in diseases and functions. These data highlight the importance of 
medRxiv preprint doi: https://doi.org/10.1101/2021.02.23.21252221; this version posted February 26, 2021. The copyright holder for this preprint (which was not certified by peer review) is the author/funder, who has granted medRxiv a license to display the preprint in perpetuity. All rights reserved. No reuse allowed without permission.

628 SARS-CoV-2 abundance in the lower airways as a predictor for mortality, and the 629 significant contribution of the host cell transcriptome, which reflects the lower airway cell 630 response to infection. 
medRxiv preprint doi: https://doi.org/10.1101/2021.02.23.21252221; this version posted February 26, 2021. The copyright holder for this preprint (which was not certified by peer review) is the author/funder, who has granted medRxiv a license to display the preprint in perpetuity.

All rights reserved. No reuse allowed without permission.

\section{Discussion}

632 A limited number of studies to date have evaluated the lower airway microenvironment

633 in patients with SARS-CoV-2 infection because of the increased risk of virus

634 transmission to healthcare providers during sampling ${ }^{19,20,37-42}$. This has limited

635 molecular investigations into the primary site of the disease. Having built a substantial

636 biorepository of lower airway samples among COVID-19 patients on mechanical

637 ventilation recruited during the first wave of SARS-CoV-2 infections in New York City,

638 we used a metagenomic approach to characterize the microbiome in the lower airways

639 and assessed its impact on clinically meaningful outcomes. In this analysis of 142

640 critically ill hospitalized patients with confirmed SARS-CoV-2 infection and lower airway

641 biorepository samples available, we determined that higher SARS-CoV-2 viral load,

642 higher relative abundance of Mycoplasma salivarium, and limited anti-SARS-CoV-2

643 Spike protein IgG response in the lower airways were associated with increased

644 mortality. This signature was supported by the metatranscriptome data of the lower

645 airway samples where SARS-CoV-2 sequence reads were significantly enriched in

646 those patients who died compared to those who survived after developing respiratory

647 failure requiring mechanical ventilation. Importantly, although we observed changes in

648 other microbial components of the lower airway microbiome in our analysis of lower

649 airway samples from 118 patients and by clinical laboratory culture results obtained

650 from 589 patients, we did not find evidence to support the hypothesis that co-infection

651 with common (bacterial, viral, fungi) respiratory pathogens was associated with poor

652 outcome-although most patients received empiric treatment with broad spectrum

653 antibiotics and anti-fungals.

654 
655 Several studies have explored the relationship between SARS-CoV-2 viral load and

656 mortality ${ }^{43-48}$. Severe influenza requiring hospitalization has also been associated with

657 higher viral loads ${ }^{49,50}$. It has been argued that high viral load might merely be a 658 reflection of an individual's immune response ${ }^{43}$. In fact, in SARS-CoV-1, clinical

659 progression was not associated with increased viral load or uncontrolled viral replication

660 in the nasopharynx but rather with an upregulated immune profile in these patients ${ }^{51}$. In

661 a large cohort of 1145 patients with confirmed SARS-CoV-2, viral load measured in

662 nasopharyngeal swab samples was found to be significantly associated with mortality,

663 even after adjusting for age, sex, race and several co-morbidities ${ }^{48}$. Similar results were

664 found in a cohort of patients in New York City with or without cancer, where in-hospital

665 mortality was significantly associated with a high SARS-CoV-2 viral load in the upper

666 airways ${ }^{47}$. The data presented here through the use of direct quantitative methods

667 (RT-PCR) and a semiquantitative untargeted approach (metatranscriptome sequencing)

668 support the hypothesis that the SARS-CoV-2 viral load in the lower airways plays a

669 critical role in the clinical progression of critically ill COVID-19 patients. It is important to

670 note that current guidelines for treatment of COVID-19 do not recommend treatment

671 with remdesivir for patients receiving invasive mechanical ventilation ${ }^{52}$. The results of

672 this investigation suggest that antivirals might still have a role in the treatment of

673 critically ill COVID-19 patients.

674

675 We investigated the possibility that mortality with SARS-CoV-2 infection was related to

676 co-infection with other pathogens. To this point several investigations have shown

677 evidence of SARS-CoV-2 co-infection with other viruses, bacteria and fungi identified by

678 culture-based techniques ${ }^{18,53-60}$. In a cohort of 116 specimens positive for SARS-CoV- 
$6792,21 \%$ were positive for one or more additional respiratory pathogens including

680 rhinovirus/enterovirus and respiratory syncytial virus ${ }^{53}$. In a meta-analysis of 3,338

681 patients with COVID-19, only $3.5 \%$ of patients had an identified bacterial co-infection at

682 admission, while $14.3 \%$ were found later to have a secondary bacterial infection ${ }^{55}$. The

683 most common pathogens identified included species in the genera Mycoplasma,

684 Hemophilus, and Pseudomonas. In another study, the most commonly identified co-

685 infections were with Streptococcus pneumoniae, Klebsiella pneumoniae, and

686 Haemophilus influenzae ${ }^{57}$. Using detailed clinical laboratory culture data available for

687589 subjects hospitalized with respiratory failure due to COVID-19, we showed that

688 higher rates of respiratory infection with other organisms, especially early in their

689 hospitalization, did not occur among subjects with poor clinical outcome. Further, we did

690 not observe an association between positive cultures for any pathogen tested and

691 increased odds of dying in critically ill COVID-19 patients.

692

693 In the subset of COVID-19 patients with BAL samples, we used NGS to identify all

694 potential pathogens and commensals in the lower airways beyond microbial cultures

695 routinely obtained as per clinical care. The RNA virome data showed that SARS-CoV-2

696 dominates the lower airways and was significantly associated with death. A small

697 number of samples had a few sequences that mapped to influenza $A$ or B viruses,

698 suggesting that co-infection with influenza did not occur frequently during this first wave

699 of SARS-CoV-2 infections. Within the DNA virome, there was no significant difference in

700 viruses between the three outcome groups despite the frequent finding of $\mathrm{HSV}-1$.

701 Similarly, when evaluating the metatranscriptome of DNA viruses, there were few

702 differences between the three outcome groups. Although analysis of the phage 
703 metagenome data showed no differential enrichment between the three cohorts, we did

704 identify in the metatranscriptome data differentially active phages when comparing the

705 three cohorts, suggesting that changes in the bacterial microbiome may be occurring in

706 critically ill patients with COVID-19. Certain Staphylococcus phages were differentially

707 active in those who were ventilated for more than 28 days and in those who died.

708 Interestingly, the bacterial signatures also identified Mycoplasma salivarium, a known

709 oral commensal that has previously been associated with ventilator-acquired

710 pneumonia ${ }^{61}$, as differentially active in those who died and those who were ventilated

711 for more than 28 days when compared to those ventilated less than 28 days. From

712 previous data published by us, enrichment of the lower airway microbiota with oral

713 commensals was seen to be associated with a pro-inflammatory state in several

714 diseases including lung cancer ${ }^{62,63}$ and non-tuberculosis mycobacterium related

715 bronchiectasis ${ }^{64}$.

717 With the use of metagenomic and metatranscriptomic analyses it is also possible to

718 examine how functionally active microbes impact the host ${ }^{65}$. In this cohort of patients,

719 we evaluated the functional profile of the microbiome within the lower airways and its

720 effect on mortality, something that, to our knowledge, had not yet been assessed in

721 COVID-19 patients. The only significant gene function enrichment was found with the

722 metatranscriptome data suggesting that functional activation of microbes can provide

723 further insights into the lower airway microbial environment of patients with worst

724 outcome. Among the pathways that were differentially expressed in those patients with

725 poor outcome, we identified genes associated with degradation, transport, and

726 antimicrobial resistance genes, as well as with signaling. These differences may 
727 indicate important functional differences leading to a different metabolic environment in

728 the lower airways that could impact host immune responses. It could also be

729 representative of differences in microbial pressure in patients with higher viral loads and

730 different inflammatory environments.

732 In the current investigation, we also characterized the immune response within the 733 lower airways by measuring anti-SARS-CoV-2 Spike antibodies and profiling the host

734 RNA transcriptome. We observed that low levels of anti-Spike and anti-RBD IgG in the

735 lung were associated with poor outcome. Although we did not find a statistically

736 significant association between SARS-CoV-2 neutralizing capacity and poor outcome,

737 levels of SARS-CoV-2 neutralizing antibodies, anti-Spike and anti-RBD antibodies (both

$738 \lg$ and $\lg$ A) were negatively correlated with SARS-CoV-2 viability. Prior investigations

739 have suggested that IgA levels are a key driver of neutralization in the mucosa ${ }^{66-68}$. The

740 differences noted in the current investigation in the $\lg$ g pools are intriguing and future

741 work investigating the antibodies generated during SARS-CoV-2 infections will be

742 essential.

744 When examining host transcriptomic differences across the different clinical outcome 745 groups, Sirtuin and Ferroptosis signaling pathways were found to be upregulated in the 746 most critically ill COVID-19 patients. Upregulation in the Sirtuin pathway demonstrates

747 an increased host inflammatory response to viral infection ${ }^{23}$. In addition, ferroptosis, a

748 recently identified form of non-apoptotic regulated cell death through iron-dependent

749 accumulation of lipid peroxides, has been shown to cause direct lung injury ${ }^{69}$ or

750 pulmonary ischemia-reperfusion injury ${ }^{70,71}$. Interestingly, there is evidence to support 
751 that STAT3 $^{69}$ and ACSL4 ${ }^{70}$ alleviated ferroptosis-mediated acute lung injury

752 dysregulation, which are both down-regulated in COVID-19 patients with worse clinical

753 outcome. Further analysis showed that there appeared to be an inactivation of

754 phagocytes, neutrophils, granulocytes, and leukocytes, including downregulation of IgG

755 expression levels, with additional mitochondria dysfunction, and down-regulation of

756 Inositol related pathways and noradrenaline/adrenaline degradation. There is evidence

757 that in the neonatal lung, inositol related components exert an anti-inflammatory effect

758 and can prevent acute lung injury ${ }^{72,73}$.

760 Collectively, these data suggest that an imbalance rather than an elevated inflammatory

761 state in the lung is an important marker that predicts poor outcomes in critically ill

762 COVID-19 patients. Indeed, the inferred cell composition analysis from the bulk

763 transcriptome data overall points to a tepid immune response. Memory $\mathrm{T}$ cells have

764 been implicated with a robust immune response in SARS-CoV-2. ${ }^{74}$ The deficiency of

765 these memory $T$ cells that we found in the lungs of COVID-19 patients with worse

766 outcome further supports the presence of an ineffective immune response or presence

767 of immune exhaustion. IL4I1, found in the network analysis to be up-regulated in the

768 deceased group in association with SARS-CoV-2, is an immunosuppression enzyme

769 that plays a role in infection and the control of immunopathology ${ }^{75}$. IL4I1 induction has

770 been reported in viral infections with influenza virus ${ }^{76}$. The ISGs and transcriptional

771 regulators $S P 110$ and SP140, both downregulated in the deceased group. play

772 important roles in resisting intracellular pathogens ${ }^{77}$. 
774 Strikingly, interrogation of the host transcriptomic analysis identified survival-associated

775 differences in interferon-related responses. Our host transcriptomic risk stratified model

776 seems to point to a predictive activation of type I interferon as a prediction for mortality.

777 This might be inconsistent with the current suggestion that, based on systemic levels,

778 early interferon responses are associated with poor outcome in COVID19. ${ }^{78,79}$ Others

779 have suggested that a robust interferon response may lead to a hyperinflammatory

780 state that could be detrimental in the disease process, justifying the use of Janus kinase

781 inhibitor inhibitors in patients with COVID-19. ${ }^{80}$ Studies comparing transcriptomic

782 signatures in BAL of patients with severe COVID-19 and controls have shown activation

783 of type 1 interferons.$^{81}$ While further longitudinal data will be needed to clarify the role of

784 interferon signaling on the disease, the data presented here suggest that combining

785 microbial and host signatures could help understand the increase risk for mortality in

786 critically ill COVID-19 patients.

788 By collecting BAL samples rather than endotracheal aspirate specimens we were able

789 to ensure extensive sampling of the lower respiratory tract in intubated patients.

790 However,

791 we were limited to samples from intubated patients in whom a clinically indicated

792 bronchoscopy was done to place a percutaneous tracheostomy or for airway clearance.

793 Although this included a large number of patients with various clinical outcomes, those

794 sampled may not be representative of the extremes in the spectrum of disease severity

795 who were most likely not eligible for bronchoscopy. For example, patients that 796 presented with very rapid clinical deterioration and died within the first few days of

797 hospitalization or those who were quickly weaned from mechanical ventilation did not 
798 receive bronchoscopy. However, extensive and detailed clinical data were also obtained

799 from intubated COVID-19 patients without bronchoscopy performed within the

800 Manhattan Campus (no bronchoscopy cohort) and from the Long Island cohort for

801 whom bronchoscopies were done without collecting research samples. In both of these

802 cohorts, clinical laboratory culture data did not identify untreated secondary pathogen

803 infections associated with poor outcome.

804

805 The samples used in this investigation were obtained during the first surge of cases of

806 COVID-19 in New York City, and management reflected clinical practices at that time.

807 Among the differences with current therapeutic approaches in COVID-19 patients,

808 corticosteroids and remdesivir, two medications that likely affect the lower airway

809 microbial landscape, were rarely used during the first surge. Other medications, such as

810 antibiotics and anti-inflammatory drugs could affect our findings and we therefore

811 considered them as potential confounders. However, the use of these medications was

812 not found to be associated with clinical outcome. The cross-sectional study design

813 precluded evaluation of the temporal dynamics of the microbial community or the host

814 immune response in this cohort, which could provide important insights into the

815 pathogenesis of this disease. Performing repeated bronchoscopies without a clinical

816 indication would be challenging in these patients and other less invasive methods might

817 need to be considered to study the lower airways at earlier timepoints and serially over

818 time in patients with respiratory failure. It is important to note that there were no

819 statistically significant differences in the timing of sample collection across the three 820 outcome groups. 
822 In summary, we present here the first evaluation of the lower airway microbiome using a

823 metagenomic and metatranscriptomic approach, along with host immune profiling in

824 critically ill patients with COVID-19 requiring invasive mechanical ventilation. The RNA

825 metatranscriptome analysis showed an association between the abundance of SARS-

826 CoV-2 and mortality, consistent with the signal found when viral load was assessed by

827 targeted rRT-PCR. These viral signatures correlated with lower anti-SARS-CoV-2 Spike

828 IgG and host transcriptomic signatures in the lower airways associated with poor

829 outcome. Importantly, both through culture and NGS data, we did not find evidence for

830 an association between untreated infections with secondary respiratory pathogens and

831 mortality. Together, these data suggest that active lower airway SARS-CoV-2

832 replication and poor SARS-CoV-2-specific antibody responses are the main drivers of

833 increased mortality in COVID-19 patients requiring mechanical ventilation. The potential

834 role of oral commensals such as Mycoplasma salivarium need to be explored further. It

835 is possible that $M$. salivarium can impact key immune cells and has recently been

836 reported at a high prevalence in patients with ventilator-acquired pneumonia ${ }^{61}$. Critically,

837 our finding that SARS-CoV-2 evades and/or derails effective innate/adaptive immune

838 responses indicates that therapies aiming to control viral replication or induce a targeted

839 antiviral immune response may be the most promising approach for hospitalized

840 patients with SARS-CoV-2 infection requiring invasive mechanical ventilation. 


\section{Methods}

\section{Subjects}

845 Enrolled subjects were 18 years or older, admitted to the intensive care units (ICUs) at

846 NYU Langone Health from March $10^{\text {th }}$ to May $10^{\text {th }}, 2020$ with a nasal swab confirmed

847 diagnosis of SARS-CoV-2 infection by reverse transcriptase polymerase chain reaction

848 (RT-PCR) assay and respiratory failure requiring invasive mechanical ventilation.

849 Samples were obtained during clinically indicated bronchoscopy performed for airway

850 clearance or for percutaneous tracheostomy placement. Surviving subjects signed

851 informed consent to participate in this study. Samples and metadata from subjects who

852 died or were incapacitated were de-identified and included in this study. Comprehensive

853 demographic and clinical data were collected. We also collected longitudinal data on

854 clinical laboratory culture results and treatment. Supplementary figure 1 shows the

855 distribution of subjects and sampling strategy used for this study. The study protocol

856 was approved by the Institutional Review Board of New York University.

\section{Lower airway bronchoscopic sampling procedure}

859 Both background and supraglottic (buccal) samples were obtained prior to the

860 procedure, as previously described ${ }^{62}$. The background samples were obtained by

861 passing sterile saline through the suctioning channel of the bronchoscope prior to the

862 procedure. Bronchoalveolar lavage (BAL) samples were obtained from one lung

863 segment as per discretion of the treating physician as clinically indicated. Samples were

864 then transferred to a BSL3 laboratory for processing. Once there, $2 \mathrm{~mL}$ of whole BAL

865 was stored in a tube prefilled with $2 \mathrm{~mL}$ of Zymo Research's DNA/RNA Shield ${ }^{\mathrm{TM}}$

866 (R1100-250, https://www.zymoresearch.com/pages/covid-19-efforts) for RNA/DNA 
867 preservation and virus inactivation. In addition, background control samples (saline

868 passed through the bronchoscope prior to bronchoscopy) and supraglottic aspirates

869 were stored in the same RNA/DNA shield. A subset of samples underwent BAL cell

870 separation by centrifugation and cells were cryopreserved in DMSO while acellular BAL

871 fluid was aliquoted for cytokine measurements. A paired blood sample was also

872 obtained in EDTA tubes (Becton Dickinson, ref\# 366450) and PAXgene Blood RNA

873 tubes (PreAnalytiX) ref\# 762165).

$875 \quad$ Viral load detection targeting the $N$ gene

876 SARS-CoV-2 viral load was measured by quantitative real-time reverse transcription

877 polymerase chain reaction (rRT-PCR) targeting the SARS-CoV-2 nucleocapsid (N)

878 gene and an additional primer/probe set to detect the human RNase $\mathrm{P}$ gene (RP).

879 Assays were performed using Thermo Fisher Scientific (Waltham, MA) TaqPath 1-Step

880 RT-qPCR Master Mix, CG (catalog number A15299) on the Applied Biosystems (Foster

881 City, CA) 7500 Fast Dx RealTime PCR Instrument. Using the positive controls provided

882 by the CDC, which are normalized to 1000 copies $/ \mathrm{mL}$, we converted the different Ct

883 positive to copies/mL. This was done using the DDCT method, applying the formula:

884 Power [2, (CT (sample, N1 gene) - CT (PC, N1 gene)] - [CT (sample, RP gene) - CT

885 (PC, RP gene)]¹000.

887 SARS-CoV-2 viral viability through measurement of subgenomic transcripts

888 Viral subgenomic mRNA (sgRNA) is transcribed in infected cells and is not packaged

889 into virions. Thus, presence of sgRNA is indicative of active infection of a mammalian 
890 cell in samples. We therefore measure sgRNA in all BAL samples obtained targeting the

891 E gene as previously described. ${ }^{21}$ Briefly, five $\mu$ RNA was used in a one-step real-time

892 RT-PCR assay to sgRNA (forward primer 5'- CGATCTCTTGTAGATCTGTTCTC-3';

893 reverse primer 5'- ATATTGCAGCAGTACGCACACA-3'; probe 5'-FAM-

894 ACACTAGCCATCCTTACTGCGCTTCG-ZEN-IBHQ-3') and using the Quantifast Probe

895 RT-PCR kit (Qiagen) according to instructions of the manufacturer. In each run, 896 standard dilutions of counted RNA standards were run in parallel to calculate copy 897 numbers in the samples.

900 DNA and RNA were isolated in parallel using zymoBIOMICS ${ }^{\mathrm{TM}}$ DNA/RNA Miniprep Kit

901 (Cat: R2002) as per manufacturer's instructions. DNA was then used for whole genome

902 shotgun (WGS) sequencing using it as input into the NexteraXT library preparation kit

903 following the manufacturer's protocol. Libraries were purified using the Agencourt

904 AMPure XP beads (Beckman Coulter, Inc.) to remove fragments below 200 bp. The

905 purified libraries were quantified using the Qubit dsDNA High Sensitivity Assay kit

906 (Invitrogen) and the average fragment length for each library was determined using a

907 High Sensitivity D1000 ScreenTape Assay (Agilent). Samples were added in an

908 equimolar manner to form two sequencing pools. The sequencing pools were quantified

909 using the KAPA Library Quantification Kit for Illumina platforms. The pools were then

910 sequenced on the Illumina Novaseq 6000 in one single run. For RNA sequencing, RNA

911 quantity and integrity were tested with a BioAnalyzer 2100 (Agilent). Among

912 bronchoscope control (BKG) samples, only 5 yielded RNA with sufficient quality and

913 quantity to undergo library preparation and sequencing. The automated Nugen Ovation 
medRxiv preprint doi: https://doi.org/10.1101/2021.02.23.21252221; this version posted February 26, 2021. The copyright holder for this preprint (which was not certified by peer review) is the author/funder, who has granted medRxiv a license to display the preprint in perpetuity.

All rights reserved. No reuse allowed without permission.

914 Trio Low Input RNA method was used for library prep with 3ng total RNA input of each

915 sample. After 6 amplification cycles, samples were sequenced using 2x Novaseq 6000

916 S4 200 cycle Flowcells using PE100 sequencing.

917

918 Microbial community characterization using whole genome shotgun sequencing (WGS)

919 and RNA metatranscriptome

920 For all metagenomic and metatranscriptomic reads, Trimmomatic v0.36 ${ }^{82}$, with leading

921 and trailing values set to 3 and minimum length set to 36 , was used to remove adaptor

922 sequences. All rRNA reads were then removed from the metatranscriptomic reads using

923 SortMeRNA v4.2.0 $0^{83}$ with default settings. Metagenomic and filtered metatranscriptomic

924 reads were mapped to the human genome using Bowtie2 v2.3.4. ${ }^{84}$ with default settings

925 and all mapping reads were excluded from subsequent microbiome, mycobiome, and

926 virome metagenomic and metatranscriptomic analysis. Technical replicates for each

927 biological sample were pooled together for subsequent analyses. Taxonomic profiles for

928 all metagenomic and metatranscriptomic samples were generated using Kraken

929 v2.0.7 $7^{85}$ and Bracken v2.5 [https://doi.org/10.7717/peerj-cs.104] run with default

930 settings. The database used for quantifying taxonomic profiles was generated using a

931 combined database containing human, bacterial, fungal, archaeal, and viral genomes

932 downloaded from NCBI RefSeq on January 8, 2021. Additionally, genomes for Candida

933 auris (Genbank: GCA_003013715.2, GCA_008275145.1) and Pneumocystic jirovecii

934 (Genbank: GCA_001477535.1) were manually added to the database. Differentially

935 abundant bacterial and viral taxa were identified for the BAL and UA samples groups

936 individually using DESeq2 v1.28.1 ${ }^{86}$ with the three group clinical outcome meta-data

937 readouts set as the sample groupings. Significantly differentially abundant taxa 
938 contained at a minimum an aggregate of 5 reads across samples and had an FDR

$939<0.2^{87,88}$.

941 For functional microbial profiling, processed sequencing reads were further depleted of

942 human-mapping reads by removing all reads classified as human by Kraken v2.0.7

943 using KrakenTools v0.1-alpha (https://github.com/ienniferlu717/KrakenTools). FMAP

$944 \mathrm{v} 0.15^{89}$ was run on both the metagenomic and metatranscriptomic reads to profile the

945 metabolic pathways present in each sample. FMAP_mapping.pl paired with diamond

946 v0.9.24 ${ }^{90}$ and FMAP_quantification.pl were used with default settings to identify and

947 quantify proteins in the Uniref90 database. Using DESeq2 v1.28.1 ${ }^{86}$, differentially

948 expressed genes were identified for the BAL samples individually using the three group

949 clinical outcome-metadata readouts for all genes that had an aggregate 5 reads across

950 all samples.

951

952 Antibiotic resistance genes were quantified in all metagenome and metatranscriptome

953 samples using Salmon v1.3.0 ${ }^{91}$ run with --keepDuplicates for indexing and --libtype A --

954 allowDovetail --meta for quantification. Genes were filtered such that only genes that

955 actively conferred antibiotic resistance were kept. To assess differentially expressed

956 classes of antibiotic resistance genes, gene counts for individual antibiotic resistance

957 genes were collapsed by their conferred antibiotic resistance.

958

959 Supplementary Figure 1 shows a summary of depth achieved with the parallel WGS 960 and metatranscriptome approach across sample types and the number of reads 961 assigned to different microbial subfractions (bacteria, fungi, DNA viruses, RNA viruses 
962 and phages). Further analysis was also done to identify possible contaminants in the

963 metatranscriptome and metagenome datasets. To this end, we compared the relative

964 abundance of taxa between background bronchoscope control and BAL samples. Taxa

965 with median relative abundance greater in background than in BAL were identified as

966 probably contaminant and listed in Supplementary Table 4). None of the taxa identified

967 as possible contaminants were removed from the analyzed data but are shown for 968 comparison with signatures identified in the rest of the analyses.

971 Anti-Spike SARS-CoV-2 antibody profiling in BAL

972 BAL samples were heat-treated at $56^{\circ} \mathrm{C}$ for one hour, and centrifuged at $14000 \mathrm{~g}$ for 5

973 min. The supernatant was collected and diluted 50 -fold in PBST containing $1 \%$ skim

974 milk. The diluted samples were incubated at room temperature (R.T.) for 30 min with

975 QBeads DevScreen: SAv (Streptavidin) (Sartorius 90792) that had been loaded with

976 biotinylated Spike, biotinylated RBD or biotin (negative control) in wells of a 96 well HTS

977 filter plate (MSHVN4550). As positive controls, we used CR3022 antibody, that

978 recognizes SARS-CoV-2 Spike and RBD, in human IgG, IgA and IgM formats (Absolute

979 Antibody). After washing the beads, bound antibodies were labeled with anti IgG-

980 DyLight488, anti IgA-PE and anti IgM-PECy7, and the fluorescence intensities were

981 measured in Intellicyt IQue3 (Sartorius). The acquired data [median fluorescence

982 intensity (MFI)] were normalized using the MFI values of the CR3022 antibodies to

983 compensate for variations across plates. Supplementary Figure 10 shows that the

984 levels of these antibodies were higher in BAL samples of patients with SARS-CoV-2

985 than in BAL samples from 10 uninfected healthy smokers recruited for research 
986 bronchoscopy. Details of method development and validation will be described

987 elsewhere (Koide et al. in preparation).

988

989 SARS-CoV-2 preparation and neutralization assay

990 icSARS-CoV-2-mNG (isolate USAWA/1/2020, obtained from the UTMB World

991 Reference Center for Emerging Viruses and Arboviruses) was amplified once in Vero E6

992 cells (P1 from the original stock). Briefly, 90-95\% confluent T175 flask (Thomas

993 Scientific) of Vero E6 ( $1 \times 10^{7}$ cells) was inoculated with $50 \mu \mathrm{L}$ of icSARS-CoV-2-mNG in

$9945 \mathrm{~mL}$ of infection media (DMEM, 2\% FBS, 1\% NEAA, and $10 \mathrm{mM}$ HEPES) for 1 hour.

995 After 1 hour, $20 \mathrm{~mL}$ of infection media was added to the inoculum and cells were

996 incubated 72 hours at $37^{\circ} \mathrm{C}$ and $5 \% \mathrm{CO}_{2}$. After 72 hours, the supernatant was collected

997 and the monolayer was frozen and thawed once. Both supernatant and cellular fractions

998 were combined, centrifuged for $5 \mathrm{~min}$ at $1200 \mathrm{rpm}$, and filtered using a $0.22 \mu \mathrm{m}$ Steriflip

999 (Millipore). Viral titers were determined by plaque assay in Vero E6 cells. In brief,

1000220,000 Vero E6 cells/well were seeded in a 24 well plate, 24 hours before inoculation.

1001 Ten-fold dilutions of the virus in DMEM (Corning) were added to the Vero E6

1002 monolayers for 1 hour at $37{ }^{\circ} \mathrm{C}$. Following incubation, cells were overlaid with $0.8 \%$

1003 agarose in DMEM containing $2 \%$ FBS (Atlanta biologicals) and incubated at $37^{\circ} \mathrm{C}$ for

$100472 \mathrm{~h}$. The cells were fixed with $10 \%$ formalin, the agarose plug removed, and plaques

1005 visualized by crystal violet staining. All procedures including icSARS-CoV-2-mNG virus

1006 were performed using Biosafety Level 3 laboratory conditions.

1007

1008 For SARS-CoV-2 neutralization assays, Vero E6 cells (30,000 cells/well) were seeded

1009 in a 96 well plate $24 \mathrm{~h}$ before infection. Two-fold serial dilutions of BAL lysates were 
1010 mixed with mixed 1:1 (vol/vol) with SARS-CoV-2 mNG virus (multiplicity of infection,

$1011 \mathrm{MOI} 0.5$ ), and incubated for $1 \mathrm{~h}$ at $37^{\circ} \mathrm{C}$. After incubation, $100 \mu \mathrm{L}$ of the mixtures of the

1012 antibody and SARS-CoV-2 mNG were added to the Vero E6 monolayers, and cells

1013 were incubated at $37^{\circ} \mathrm{C}$. After $20 \mathrm{~h}$, cells were fixed with $4 \%$ formaldehyde (Electron

1014 Microscopy Sciences) at room temperature for $1 \mathrm{~h}$. After fixation, cells were washed

1015 twice with PBS and permeabilized with $0.25 \%$ triton-100, stained with DAPI (Thermo),

1016 and quantified on a Celllnsight CX7 High-content microscope (Thermo) using a cut-off

1017 for three standard deviations from negative to be scored as an infected cell.

\section{$\underline{\text { Transcriptome of BAL cells }}$}

1020 RNA-Seq was performed on bronchial epithelial cells obtained by airway brushing, as

1021 described $^{92-94}$, using the Hi-seq/lllumina platform at the NYU Langone Genomic

1022 Technology Center (data available at Sequence Read Archive: \# PRJNA592149).

$1023 \mathrm{KEGG}^{95,96}$ annotation was summarized at levels 1 to 3 . Genes with an FDR-corrected

1024 adjusted p-value <0.25 were considered significantly differentiated, unless otherwise

1025 specified. Pathway analysis using differentially regulated genes (FDR<0.25) was done

1026 using Ingenuity Pathway Analysis, RRID:SCR_0- at least 1 count per million in at least

1027 two samples were retained. For digital cytometry with CIBERSORTx, a signature matrix

1028 derived from single-cell transcriptome of BAL cells collected from patients with COVID-

$102919^{31}$ was first generated with the "Create Signature Matrix" module in the CIBERSORTx

1030 online tool. A maximum of 10 cells per cell type per patient were initially sampled from

1031 the original data and 20 cells per cell type were then used to build the single-cell

1032 reference with the default parameters. Then the "Impute Cell Fractions" module was

1033 used to estimate the absolute cell fraction score of different cell types in bulk 
1034 transcriptomes using the single-cell signatures with "S-mode" batch correction and 100

1035 permutations in the absolute mode. Bulk transcriptomes with a significant deconvolution

1036 p-value $(\leq 0.05)$ were retained. For xCell cell type signature enrichment analysis, the

1037 enrichment scores were inferred with built-in signature of cell types detected in the BAL

1038 samples as reported previously ${ }^{31}$. The two-tailed Wilcoxon rank sum test with

1039 Benjamini-Hochberg correction were computed between groups of samples for 1040 comparison.

1041

1042 Microbial and Host predictive modeling

1043 Cox proportional hazards model was used for investigating the association between the

1044 time to death and the relative abundance of each taxon quantified using

1045 metatranscriptomic and metagenomic data separately. We first performed the univariate

1046 screening test to identify significant features associated with the time to death using the

1047 Cox proportion hazards regression model for the relative abundance of taxa from the

1048 RNA and DNA data, and log-transformed count of host transcriptome data, respectively.

1049 Within each type of data, given the $p$-value cutoff, the features with a p-value less than

1050 the cutoff were selected and integrated as a sub-community. For the RNA and DNA

1051 data, the alpha diversity (Shannon index) was calculated for each sample on the

1052 selected sub-community and the negative of the value was defined as the microbial risk

1053 score, because high alpha diversity indicates low risk of death. For the host

1054 transcriptome data, the log-transformed total count of all selected candidate

1055 transcriptome for each sample was defined as the risk score, since most selected 1056 candidate transcriptomes increased the risk of death. The leave-one-out cross1057 validation (LOOCV) was used for the predictions. The $p$ value cutoff was set at the 
1058 value which produces the largest AUC (area under the receiver operating characteristic

1059 curve) in predicting the death/survival status using the risk score we constructed over

1060 these features. The additive model was used to integrate when more than one scores

1061 are used for the prediction.

1062

1063 Multiscale and co-expression network analyses

1064 Raw counts from the human transcriptome were normalized and converted to log2-

1065 counts per million using $\operatorname{limma}^{97} / \mathrm{voom}^{98}$ (v3.44.1 with $\mathrm{R}$ v4.0.0) with standard 1066 parameters. Microbiome abundance information was converted to relative abundance.

1067 Low abundance taxa were removed based on average abundance across all samples to 1068 yield a minimum of 1000 taxa for each metatranscriptome dataset. All datasets were 1069 batch adjusted. Differentially expressed genes (DEGs) and differentially abundant taxa 1070 were called using the DESeq2 package ${ }^{86}$ (v1.28.1), based on the negative binomial (i.e.

1071 Gamma-Poisson) distribution. According to the recommendation by the authors, we

1072 used non-normalized data (i.e. raw gene counts and abundance data), as DESeq2 1073 internally corrects data and performs normalization steps. For this purpose, raw 1074 microbiome abundance data were converted to DESeq2 dds objects using the phyloseq 1075 R library (V1.32.0). Contrasts are based on outcome groups ( $\leq 28$ days MV, $>28$ days 1076 MV or death). Differentially expressed genes and differentially abundant tax with FDR of $1077 \quad 0.2$ or less are considered significant.

1078 Multiscale Embedded Gene Co-Expression Network Analysis (MEGENA) ${ }^{32}$ was 1079 performed to identify host modules of highly co-expressed genes in SARS-CoV-2 1080 infection. The MEGENA workflow comprises four major steps: 1) Fast Planar Filtered 1081 Network construction (FPFNC), 2) Multiscale Clustering Analysis (MCA), 3) Multiscale 
1082 Hub Analysis (MHA), 4) and Cluster-Trait Association Analysis (CTA). The total

1083 relevance of each module to SARS-CoV-2 infection was calculated by using the Product

1084 of Rank method with the combined enrichment of the differentially expressed gene

1085 (DEG) signatures as implemented: $G_{j}=\prod_{i} g_{j i}$, where, $g_{j i}$ is the relevance of a

1086 consensus $\boldsymbol{j}$ to a signature $\boldsymbol{i}$; and $g_{j i}$ is defined as $\left(\max _{j}\left(r_{j i}\right)+1-r_{j i}\right) / \sum_{j} r_{j i}$, where $r_{j i}$

1087 is the ranking order of the significance level of the overlap between the module $j$ and the

1088 signature.

1089

1090 To functionally annotate gene signatures and gene modules derived from the host 1091 transcriptome data, we performed an enrichment analysis of the established pathways 1092 and signatures_including the gene ontology (GO) categories and MSigDB. The hub 1093 genes in each subnetwork were identified using the adopted Fisher's inverse Chi1094 square approach in MEGENA; Bonferroni-corrected p-values smaller than 0.05 were set 1095 as the threshold to identify significant hubs. The correlation between modules, modules 1096 and clinical traits as well as modules and individual taxa were performed using

1097 Spearman correlation. Other correlation measures, such as Pearson correlation or the 1098 Maximal Information Coefficient $(\mathrm{MIC})^{99}$ proved to be inferior for this task. Categorical 1099 trait data was converted to numerical values as suitable.

$1101 \quad$ Data availability

1102 Sequencing data are available in NCBI's Sequence Read Archive under project 1103 numbers PRJNA688510 and PRJNA687506 (RNA and DNA sequencing, respectively). 1104 Codes used for the analyses presented in the current manuscript are available at 1105 https://github.com/segalmicrobiomelab/SARS_CoV2. 
medRxiv preprint doi: https://doi.org/10.1101/2021.02.23.21252221; this version posted February 26, 2021. The copyright holder for this preprint (which was not certified by peer review) is the author/funder, who has granted medRxiv a license to display the preprint in perpetuity.

All rights reserved. No reuse allowed without permission.

1106 


\section{Figure Legends:}

1109 Figure 1. Associations between culture positivity and clinical outcome. Odds

1110 ratios and corresponding 95\% confidence intervals for rates of culture positivity for the

1111 whole cohort $(n=589)$ during the length of their hospitalization (left) and during the first 2

1112 weeks of hospitalization (right).

1113

1114 Figure 2. SARS-CoV-2 viral load and virus metatranscriptome analyses. Copies of

1115 the SARS-CoV-2 $\mathrm{N}$ gene per $\mathrm{ml}$, normalized by the Human RNase P gene, comparing

1116 paired upper and lower airway samples (a) and levels in BAL comparing clinical

1117 outcome groups (b, ${ }^{*}=$ Mann-Whitney $U p<0.05,{ }^{* *}=$ Mann-Whitney $\left.U p<0.01\right)$. (c)

1118 PCoA analysis based on Bray Curtis Dissimilarity index of BAL Metatranscriptome data

1119 comparing clinical outcome (PERMANOVA p-value). Bubble plot showing DESeq

1120 results of RNA viruses (d) and expressed DNA phages (e) enriched in each clinical

1121 outcome comparisons (bubble size based on median relative abundance for those

1122 found statistically significant).

1124 Figure 3. Bacteria load and taxonomic compositional analyses. (a) Bacterial load

1125 measured by ddPCR targeting $16 \mathrm{~S}$ rRNA gene $\left({ }^{* *}=\right.$ Mann-Whitney $\left.U \mathrm{p}<0.01\right)$. PCoA

1126 analysis based on Bray Curtis Dissimilarity index of BAL Metagenome (b) and

1127 Metatranscriptome (c) data comparing clinical outcome (PERMANOVA p-value). (d)

1128 Gene Set Enrichment Analysis (GSEA) was used to compare the taxonomic signatures

1129 identified in BAL metagenome (diamonds) and metatranscriptome (circles) as distinctly

1130 enriched for comparisons between clinical outcome groups (differential enrichment 
1131 performed based on DESeq2 analysis). (e) Bubble plot showing DESeq results of

1132 bacteria found concordantly differentially enriched between clinical outcome groups

1133 (bubble size based on median relative abundance for those found statistically 1134 significant).

1136 Figure 4. Functional microbial compositional analyses. KOs were summarized to 1137 associated pathways and differential expression was calculated based on DESeq2 1138 analysis. (a) Gene Set Enrichment Analysis (GSEA) was used to compare the functional 1139 signatures identified in BAL metagenome and metatranscriptome as distinctly enriched

1140 for comparisons between clinical outcome groups. (b) Bubble plot showing DESeq 1141 results of microbial functions found concordantly differentially enriched between clinical 1142 outcome groups (bubble size based on median relative abundance for those found 1143 statistically significant).

1145 Figure 5. Lower airway host immune profiling in severely ill COVID-19. (a) Levels 1146 of anti-SARS-CoV-2 Spike antibodies in BAL ( ${ }^{*}=$ Mann-Whitney $\left.U p<0.05\right)$. (b) Heat1147 map of canonical pathway analysis based on Ingenuity Pathway Analysis (IPA, 1148 RRID:SCR_008653) using the lower airway host transcriptome comparing clinical 1149 outcome groups. Orange shows up-regulation of pathway, blue shows down-regulation 1150 of pathway. (c) Cell type abundance quantification plots. Comparison of abundance of 1151 mast cells and neutrophils among outcome groups in the BAL fluids of critically ill 1152 patients with COVID-19. Cell type abundance was estimated from the host 1153 transcriptome with CIBERSORTx. Each dot denotes the quantification score of a 1154 sample and boxes depict median and inter-quartile range $\left({ }^{*}=\right.$ Mann-Whitney $\left.\cup p<0.05\right)$. 
1156 Figure 6. Mortality predictive power of metatranscriptome, metagenome and host

1157 transcriptome. (a) Area under the curved median and confidence interval for receiver

1158 operating characteristic curve analyses calculated from each sequencing datasets as

1159 predictor and mortality as outcome. (b) Kaplan-meier survival analyses based on a

1160 cutoff value estimated from features selected from each sequencing dataset. The "High

1161 risk" and "Low risk" groups is the mean of predicted risk scores in all samples. (c)

1162 Scatterplot among risk scores from metatranscriptome, metagenome, and host

1163 transcriptome. Dotted line denotes the mean of the risk scores across all subjects,

1164 which is also the threshold for dividing the samples into "High risk" and "Low risk"

1165 groups. (d) IPA analyses of host transcriptomic signatures identified as most predictive 1166 of mortality. 


\section{Supplementary Figure Legends:}

1172 Supplementary Figure 1. Description of patient cohort, samples obtained, 1173 analyses performed and sequencing depth.

1175 Supplementary Figure 2. SARS-CoV-2 viral load in upper airway samples. Copies 1176 of the SARS-CoV-2 N gene per ml, normalized by the Human RNase P gene, in upper

1177 airways comparing clinical outcome groups (Mann-Whitney U p-value).

1180 as compared with BAL and upper airway samples. Boxplots showing the relative

1181 abundance values in $\log 10$ relative abundance of taxa ranked ordered based on

1182 dominance of Background bronchoscope control samples and compared to abundances

1183 in BAL and Upper Airway samples within metatranscriptome (a) and metagenome (b)

1184 data. Red labels indicate taxa where relative abundance is higher in background 1185 samples than in BAL and therefore considered possible contaminant.

1187 Supplementary Figure 4. Topographical analyses of Metatranscriptome data.

1188 Comparison of alpha diversity (Shannon Index, a) and beta diversity (Bray Curtis

1189 Dissimilarity index, b) across background negative controls (bronchoscope),

1190 bronchoalveolar lavage (BAL) and upper airway (UA) samples (Kruskal-Wallis and

1191 PERMANOVA p-values, respectively). (c) Boxplots showing the relative abundance

1192 values in log10 across all metatranscriptome samples for the BAL and Upper Airway 1193 samples. The 50 taxa with the highest relative abundance values in the BAL 1194 metatranscriptome data are displayed; the top 10 in the BAL are highlighted in bold. 
1195 Each column consists of four plots displaying in order from top to bottom, the most

1196 abundant RNA vertebrate viruses, DNA phages, bacteria, and fungi identified (from top

1197 to bottom). Numbers in parentheses next to the taxa labels display the ranking in

1198 relative abundance for either the BAL or UA metatranscriptome samples, respectively.

1200 Supplementary Figure 5. Topographical analyses of Metagenome Data.

1201 Comparison of alpha diversity (Shannon Index, a) and beta diversity (Bray Curtis

1202 Dissimilarity index, b) across background negative controls (bronchoscope),

1203 bronchoalveolar lavage (BAL) and upper airway (UA) samples (Kruskal-Wallis and

1204 PERMANOVA p-values, respectively). (c) Boxplots showing the relative abundance

1205 values in log10 across all metagenome samples for the BAL and Upper Airways. The 50

1206 taxa with the highest relative abundance values in the BAL metagenome are displayed;

1207 the top 10 in the BAL are highlighted in bold. Each column consists of two plots 1208 displaying the most abundant bacteria and fungi identified. Numbers in parentheses

1209 next to the taxa labels displays its ranking in relative abundance for either the BAL or

1210 UA metagenome samples, respectively.

1212 Supplementary Figure 6. Evaluation of associations between the lower airway

1213 RNA virome and clinical outcome. Comparisons between the three clinical outcome

1214 groups was performed for $\alpha$ diversity (Shannon Index, Kruskal-Wallis p-value, left

1215 panel), $\beta$ diversity (based on Bray Curtis Dissimilarity Index, PERMANOVA p-value, 1216 right panel). 
1218 Supplementary Figure 7. Topographical analyses of the bacterial load. Bacterial

1219 load measured by ddPCR targeting 16S rRNA gene in background bronchoscope

1220 controls (BKG), lower airway (BAL) and upper airway (UA) samples.

1222 Supplementary Figure 8. Evaluation of associations between the lower airway

1223 mycobiome and clinical outcome. Fungal taxonomic data was subtracted from

1224 metagenome and metatranscriptome data from lower airway samples. (a) Comparisons

1225 between the three clinical outcome groups was performed for $\alpha$ diversity (Shannon

1226 Index, Kruskal-Wallis $p$-value, left panel), $\beta$ diversity (based on Bray Curtis Dissimilarity

1227 Index, PERMANOVA p-value, right panel) on metagenome data. (b) Bubble plot

1228 showing DESeq results of fungi enriched in each clinical outcome comparisons based

1229 on metagenome data (bubble size based on median relative abundance for those found

1230 statistically significant). (c) Comparisons between the three clinical outcome groups was

1231 performed for $\alpha$ diversity (Shannon Index, Kruskal-Wallis $p$-value, left panel), $\beta$ diversity

1232 (based on Bray Curtis Dissimilarity Index, PERMANOVA p-value, right panel) on

1233 metatranscriptome data. (d) Bubble plot showing DESeq results of fungi enriched in

1234 each clinical outcome comparisons based on metatranscriptome data (bubble size

1235 based on median relative abundance for those found statistically significant).

1237 Supplementary Figure 9. Evaluation of associations between the lower airway

1238 antibiotic resistance genes and clinical outcome. Bubble plot showing DESeq

1239 results of summarized categories of antibiotic resistant microbial genes taken from

1240 MEGARes for the metagenome (top) and metatranscriptome (bottom) data sets for

1241 each clinical outcome comparison (bubble size based on median relative abundance for 
1242 those found to be statistically significant). Colored bubbles indicate significantly

1243 enriched antibiotic resistance groups.

1245 Supplementary Figure 10. Measurement of anti-SARS-CoV-2 Immunoglobulin

1246 levels and neutralization activity. Levels of anti-SARS-CoV-2 Spike (a) and anti-

1247 SARS-CoV-2 receptor binding domain (RBD, b) antibodies in BAL from non SARS-

1248 CoV-2 infected smoker controls and severely ill COVID-19 intubated patients. Note that

1249 the signals for different isotypes cannot be compared because they are detected with

1250 different reagents. (c) Comparisons of levels of anti-SARS-CoV-2 RBD antibodies in

1251 BAL across subjects in different clinical outcome groups ( ${ }^{*}=$ Mann-Whitney $\left.U p<0.05\right)$.

1252 (d) Neutralizing activity in BAL samples across subjects in different clinical outcome 1253 groups.

1254

1255 Supplementary Figure 11. Evaluation for associations between the lower airway

1256 host tanscriptome and clinical outcome. (a) PCoA (based on Bray Curtis

1257 Dissimilarity Index, PERMANOVA p-value) comparing the three clinical outcome

1258 groups. (b, c, d) Volcano plot comparing lower airway host transcriptome between the

1259 three clinical outcome groups.

1261 Supplementary Figure 12. Multi-scale cross-kingdom and co-expression

1262 networks. (a) The neighborhood 5 cross-kingdom metatranscriptome network centered

1263 around SARS-CoV-2 is shown. Nodes refer to taxa, edges denote co-abundance after

1264 MEGENA. The size of the nodes indicates abundance. Taxa with large nodes are highly

1265 abundant. Node-shapes are according to the legend and refer to different microbial 
1266 kingdoms. The differential abundance of taxa in log2(fold change) between the

1267 deceased group and the $\leq 28$-day MV groups is shown by node color - red nodes are

1268 taxa abundant in the deceased group compared to the $\leq 28$-day MV group, blue colored

1269 nodes denote the opposite. (b) Modules M175 and M718 of the host transcriptome are

1270 shown. The node size refers to the absolute gene expression value. Nodes with wide

1271 node border refer to key regulators/hub genes (see Methods). The differential gene

1272 expression of taxa in log2(fold change) between the deceased group and the $\leq 28$-day

1273 MV groups is shown by node color - red nodes are up-regulated in the deceased group

1274 compared to the $\leq 28$-day MV group, blue colored nodes denote the opposite. 


\section{Reference:}

1277

1. The, L. Emerging understandings of 2019-nCoV. Lancet 395, 311 (2020).

1278 2. WHO coronavirus disease (COVID-19) dashboard. Geneva: World Health Organization, Available online: https://covid19.who.int/ (2020).

3. Rabaan, A.A., et al. SARS-CoV-2, SARS-CoV, and MERS-COV: A comparative overview. Infez Med 28, 174-184 (2020).

1282

1283

1284

1285

1286

4. Cao, X. COVID-19: immunopathology and its implications for therapy. Nat Rev Immunol 20, 269270 (2020).

5. Arentz, M., et al. Characteristics and Outcomes of 21 Critically III Patients With COVID-19 in Washington State. JAMA 323, 1612-1614 (2020). Bhatraju, P.K., et al. Covid-19 in Critically III Patients in the Seattle Region - Case Series. N Engl J

6. Bhatraju, P.K., et al. Covid-19 in
Med 382, 2012-2022 (2020).

7. Cummings, M.J., et al. Epidemiology, clinical course, and outcomes of critically ill adults with COVID-19 in New York City: a prospective cohort study. The Lancet 395, 1763-1770 (2020). 8. European Society of Anesthesiology. Analysis of COVID-19 data on numbers in intensive care
from Italy: European Society of Anaesthesiology (ESA). Vol. 2020 (2020). CoV-2 Admitted to ICUs of the Lombardy Region, Italy. JAMA 323, 1574-1581 (2020).

10. Intensive Care National Audit and Research Center UK. ICNARC report on COVID-19 in Critical Care., Vol. 2020 (2020).

11. Richardson, S., et al. Presenting Characteristics, Comorbidities, and Outcomes Among 5700
Patients Hospitalized With COVID-19 in the New York City Area. JAMA 323, 2052-2059 (2020).

12. Yang, X., et al. Clinical course and outcomes of critically ill patients with SARS-CoV-2 pneumonia in Wuhan, China: a single-centered, retrospective, observational study. Lancet Respir Med 8 , 475-481 (2020).

13. Morens, D.M. \& Fauci, A.S. The 1918 influenza pandemic: insights for the 21st century. J Infect Dis 195, 1018-1028 (2007).

14. Shieh, W.J., et al. 2009 pandemic influenza A (H1N1): pathology and pathogenesis of 100 fatal cases in the United States. Am J Pathol 177, 166-175 (2010).

15. Dickson, R.P., et al. Enrichment of the lung microbiome with gut bacteria in sepsis and the acute respiratory distress syndrome. Nat Microbiol 1, 16113 (2016).

16. Kitsios, G.D., et al. Respiratory Tract Dysbiosis Is Associated with Worse Outcomes in Mechanically Ventilated Patients. Am J Respir Crit Care Med 202, 1666-1677 (2020).

17. Dickson, R.P., et al. Lung Microbiota Predict Clinical Outcomes in Critically III Patients. Am J Respir Crit Care Med 201, 555-563 (2020).

18. Zuo, T., et al. Alterations in Fecal Fungal Microbiome of Patients With COVID-19 During Time of Hospitalization until Discharge. Gastroenterology 159, 1302-1310 e1305 (2020).

19. Chen, L., et al. RNA based mNGS approach identifies a novel human coronavirus from two individual pneumonia cases in 2019 Wuhan outbreak. Emerg Microbes Infect 9, 313-319 (2020).

20. Shen, Z., et al. Genomic Diversity of Severe Acute Respiratory Syndrome-Coronavirus 2 in Patients With Coronavirus Disease 2019. Clin Infect Dis 71, 713-720 (2020).

21. Wolfel, R., et al. Virological assessment of hospitalized patients with COVID-2019. Nature 581, 465-469 (2020).

22. Yazdi, M., Bouzari, M. \& Ghaemi, E.A. Genomic analyses of a novel bacteriophage (VB_PmiSIsfahan) within Siphoviridae family infecting Proteus mirabilis. Genomics 111, 1283-1291 (2019). 
1323 24. Dar, H.H., et al. Pseudomonas aeruginosa utilizes host polyunsaturated

1324

1325

1326

1327

1328

1329

1330

1331

1332

1333

1334

1335

1336

1337

1338

1339

1340

1341

1342

1343

1344

1345

1346

1347

1348

1349

1350

1351

1352

1353

1354

1355

1356

1357

1358

1359

1360

1361

1362

1363

1364

1365

1366

1367

1368

1369

1370

1371

phosphatidylethanolamines to trigger theft-ferroptosis in bronchial epithelium. J Clin Invest 128, 4639-4653 (2018).

25. Stoyanovsky, D.A., et al. Iron catalysis of lipid peroxidation in ferroptosis: Regulated enzymatic or random free radical reaction? Free Radic Biol Med 133, 153-161 (2019).

26. Burtscher, J., Cappellano, G., Omori, A., Koshiba, T. \& Millet, G.P. Mitochondria: In the Cross Fire of SARS-CoV-2 and Immunity. iscience 23, 101631 (2020).

27. Smati, S., et al. Relationship between obesity and severe COVID-19 outcomes in patients with type 2 diabetes: Results from the CORONADO study. Diabetes Obes Metab (2020).

28. Wargny, M., et al. Type 1 Diabetes in People Hospitalized for COVID-19: New Insights From the CORONADO Study. Diabetes Care 43, e174-e177 (2020).

29. Newman, A.M., et al. Determining cell type abundance and expression from bulk tissues with digital cytometry. Nature Biotechnology 37, 773-782 (2019).

30. Aran, D., Hu, Z. \& Butte, A.J. xCell: digitally portraying the tissue cellular heterogeneity landscape. Genome Biology 18, 220 (2017).

31. Liao, M., et al. Single-cell landscape of bronchoalveolar immune cells in patients with COVID-19. Nature Medicine 26, 842-844 (2020).

32. Song, W.M. \& Zhang, B. Multiscale Embedded Gene Co-expression Network Analysis. PLoS computational biology 11, e1004574 (2015).

33. Praest, P., et al. The influence of TAP1 and TAP2 gene polymorphisms on TAP function and its inhibition by viral immune evasion proteins. Molecular immunology 101, 55-64 (2018).

34. Sreekumar, P.G., et al. The mitochondrial-derived peptide humanin protects RPE cells from oxidative stress, senescence, and mitochondrial dysfunction. Investigative ophthalmology \& visual science 57, 1238-1253 (2016).

35. Zhou, J.y., et al. Wnt/B-catenin-mediated heat exposure inhibits intestinal epithelial cell proliferation and stem cell expansion through endoplasmic reticulum stress. Journal of cellular physiology 235, 5613-5627 (2020).

36. Liu, W., et al. LncRNA Malat1 inhibition of TDP43 cleavage suppresses IRF3-initiated antiviral innate immunity. Proceedings of the National Academy of Sciences 117, 23695-23706 (2020).

37. American Association of Otolaryngology-Head and Neck Surgery. Tracheotomy Recommendations During the Covid-19 Pandemic. Vol. 2020 (2020).

38. Sommer, D.D., et al. Recommendations from the CSO-HNS taskforce on performance of tracheotomy during the COVID-19 pandemic. J Otolaryngol Head Neck Surg 49, 23 (2020).

39. Shiba, T., Ghazizadeh, S., Chhetri, D., St John, M. \& Long, J. Tracheostomy Considerations during the COVID-19 Pandemic. OTO Open 4, 2473974X20922528 (2020).

40. Ear Nose and Throat Surgery United Kingdom. Tracheostomy guidance during the COVID-19 Pandemic. Vol. 2020 (2020).

41. Michetti, C.P., Burlew, C.C., Bulger, E.M., Davis, K.A. \& Spain, D.A. Performing tracheostomy during the Covid-19 pandemic: guidance and recommendations from the Critical Care and Acute Care Surgery Committees of the American Association for the Surgery of Trauma. Trauma Surgery \& Acute Care Open 5, e000482 (2020).

42. McGrath, B.A., et al. Tracheostomy in the COVID-19 era: global and multidisciplinary guidance. Lancet Respir Med, S2213-2600(2220)30230-30237 (2020).

43. Kelleni, M.T. SARS CoV-2 viral load might not be the right predictor of COVID-19 mortality. $J$ Infect (2020).

44. Fajnzylber, J., et al. SARS-CoV-2 viral load is associated with increased disease severity and mortality. Nat Commun 11, 5493 (2020).

45. Bitker, L., et al. Protracted viral shedding and viral load are associated with ICU mortality in Covid-19 patients with acute respiratory failure. Ann Intensive Care 10, 167 (2020). 
1372 46. Magleby, R., et al. Impact of SARS-CoV-2 Viral Load on Risk of Intubation and Mortality Among

1373

1374

1375

1376

1377

1378

1379

1380

1381

1382

1383

1384

1385

1386

1387

1388

1389

1390

1391

1392

1393

1394

1395

1396

1397

1398

1399

1400

1401

1402

1403

1404

1405

1406

1407

1408

1409

1410

1411

1412

1413

1414

1415

1416

1417

1418

1419

Hospitalized Patients with Coronavirus Disease 2019. Clin Infect Dis (2020).

47. Westblade, L.F., et al. SARS-CoV-2 Viral Load Predicts Mortality in Patients with and without Cancer Who Are Hospitalized with COVID-19. Cancer Cell 38, 661-671 e662 (2020).

48. Pujadas, E., et al. SARS-CoV-2 viral load predicts COVID-19 mortality. Lancet Respir Med 8, e70 (2020).

49. Lee, N., et al. Viral loads and duration of viral shedding in adult patients hospitalized with influenza. J Infect Dis 200, 492-500 (2009).

50. Li, C.C., et al. Correlation of pandemic (H1N1) 2009 viral load with disease severity and prolonged viral shedding in children. Emerging infectious diseases 16, 1265-1272 (2010).

51. Peiris, J.S., et al. Clinical progression and viral load in a community outbreak of coronavirusassociated SARS pneumonia: a prospective study. Lancet 361, 1767-1772 (2003).

52. https://www.covid19treatmentguidelines.nih.gov/therapeutic-management/.

53. Kim, D., Quinn, J., Pinsky, B., Shah, N.H. \& Brown, I. Rates of Co-infection Between SARS-CoV-2 and Other Respiratory Pathogens. JAMA 323, 2085-2086 (2020).

54. Kreitmann, L., Monard, C., Dauwalder, O., Simon, M. \& Argaud, L. Early bacterial co-infection in ARDS related to COVID-19. Intensive Care Med 46, 1787-1789 (2020).

55. Langford, B.J., et al. Bacterial co-infection and secondary infection in patients with COVID-19: a living rapid review and meta-analysis. Clin Microbiol Infect 26, 1622-1629 (2020).

56. Sepulveda, J., et al. Bacteremia and Blood Culture Utilization during COVID-19 Surge in New York City. J Clin Microbiol 58(2020).

57. Zhu, X., et al. Co-infection with respiratory pathogens among COVID-2019 cases. Virus Res 285, 198005 (2020).

58. Zhang, H., et al. Risks and features of secondary infections in severe and critical ill COVID-19 patients. Emerg Microbes Infect 9, 1958-1964 (2020).

59. Lansbury, L., Lim, B., Baskaran, V. \& Lim, W.S. Co-infections in people with COVID-19: a systematic review and meta-analysis. J Infect 81, 266-275 (2020).

60. Koehler, P., et al. COVID-19 associated pulmonary aspergillosis. Mycoses 63, 528-534 (2020).

61. Nolan, T.J., et al. Low-pathogenicity Mycoplasma spp. alter human monocyte and macrophage function and are highly prevalent among patients with ventilator-acquired pneumonia. Thorax 71, 594-600 (2016).

62. Tsay, J.J., et al. Airway Microbiota Is Associated with Upregulation of the PI3K Pathway in Lung Cancer. Am J Respir Crit Care Med 198, 1188-1198 (2018).

63. Tsay, J.J., et al. Lower airway dysbiosis affects lung cancer progression. Cancer Discov (2020).

64. Sulaiman, I., et al. Evaluation of the airway microbiome in nontuberculous mycobacteria disease. Eur Respir J 52(2018).

65. Zhang, X., Li, L., Butcher, J., Stintzi, A. \& Figeys, D. Advancing functional and translational microbiome research using meta-omics approaches. Microbiome 7, 154 (2019).

66. Sterlin, D., et al. IgA dominates the early neutralizing antibody response to SARS-CoV-2. Science translational medicine 13(2021).

67. Wang, Z., et al. Enhanced SARS-CoV-2 neutralization by dimeric IgA. Science translational medicine 13(2021).

68. Klingler, J., et al. Role of IgM and IgA Antibodies in the Neutralization of SARS-CoV-2. J Infect Dis (2020).

69. Qiang, Z., et al. Nrf2 and STAT3 Alleviates Ferroptosis-Mediated IIR-ALI by Regulating SLC7A11. Oxid Med Cell Longev 2020, 5146982 (2020).

70. Xu, Y., Li, X., Cheng, Y., Yang, M. \& Wang, R. Inhibition of ACSL4 attenuates ferroptotic damage after pulmonary ischemia-reperfusion. FASEB J 34, 16262-16275 (2020). 
1420 71. Dong, H., et al. Nrf2 inhibits ferroptosis and protects against acute lung injury due to intestinal ischemia reperfusion via regulating SLC7A11 and HO-1. Aging (Albany NY) 12, 12943-12959 (2020).

72. Hallman, M., Bry, K., Hoppu, K., Lappi, M. \& Pohjavuori, M. Inositol supplementation in premature infants with respiratory distress syndrome. N Engl J Med 326, 1233-1239 (1992).

73. Preuss, S., et al. Inositol-trisphosphate reduces alveolar apoptosis and pulmonary edema in neonatal lung injury. Am J Respir Cell Mol Biol 47, 158-169 (2012).

74. Sekine, T., et al. Robust T Cell Immunity in Convalescent Individuals with Asymptomatic or Mild COVID-19. Cell 183, 158-168 e114 (2020).

75. Molinier-Frenkel, V., Prévost-Blondel, A. \& Castellano, F. The IL4I1 Enzyme: A New Player in the Immunosuppressive Tumor Microenvironment. Cells 8(2019).

76. Baas, T., et al. Integrated molecular signature of disease: analysis of influenza virus-infected macaques through functional genomics and proteomics. Journal of virology 80, 10813-10828 (2006).

77. Regad, T. \& Chelbi-Alix, M.K. Role and fate of PML nuclear bodies in response to interferon and viral infections. Oncogene 20, 7274-7286 (2001).

78. Bastard, P., et al. Autoantibodies against type I IFNs in patients with life-threatening COVID-19. Science 370(2020).

79. Zhang, Q., et al. Inborn errors of type I IFN immunity in patients with life-threatening COVID-19. Science 370(2020).

80. Kalil, A.C., et al. Baricitinib plus Remdesivir for Hospitalized Adults with Covid-19. N Engl J Med (2020).

81. Zhou, Z., et al. Heightened Innate Immune Responses in the Respiratory Tract of COVID-19 Patients. Cell host \& microbe 27, 883-890 e882 (2020).

82. Bolger, A.M., Lohse, M. \& Usadel, B. Trimmomatic: a flexible trimmer for Illumina sequence data. Bioinformatics 30, 2114-2120 (2014).

83. Kopylova, E., Noe, L. \& Touzet, H. SortMeRNA: fast and accurate filtering of ribosomal RNAs in metatranscriptomic data. Bioinformatics 28, 3211-3217 (2012).

84. $\quad$ Langmead, B. \& Salzberg, S.L. Fast gapped-read alignment with Bowtie 2. Nat Methods 9, 357359 (2012).

85. Wood, D.E., Lu, J. \& Langmead, B. Improved metagenomic analysis with Kraken 2. Genome biology 20, 257 (2019).

86. Love, M.I., Huber, W. \& Anders, S. Moderated estimation of fold change and dispersion for RNAseq data with DESeq2. Genome biology 15, 550 (2014).

87. Pavel, A.B., et al. Alterations in Bronchial Airway miRNA Expression for Lung Cancer Detection. Cancer Prev Res (Phila) 10, 651-659 (2017).

88. Seumois, G., et al. Transcriptional Profiling of Th2 Cells Identifies Pathogenic Features Associated with Asthma. J Immunol 197, 655-664 (2016).

89. Kim, J., Kim, M.S., Koh, A.Y., Xie, Y. \& Zhan, X. FMAP: Functional Mapping and Analysis Pipeline for metagenomics and metatranscriptomics studies. BMC Bioinformatics 17, 420 (2016).

90. Buchfink, B., Xie, C. \& Huson, D.H. Fast and sensitive protein alignment using DIAMOND. Nat Methods 12, 59-60 (2015).

91. Patro, R., Duggal, G., Love, M.I., Irizarry, R.A. \& Kingsford, C. Salmon provides fast and biasaware quantification of transcript expression. Nat Methods 14, 417-419 (2017).

92. Mortazavi, A., Williams, B.A., McCue, K., Schaeffer, L. \& Wold, B. Mapping and quantifying mammalian transcriptomes by RNA-Seq. Nat Methods 5, 621-628 (2008).

93. Wilhelm, B.T., et al. Dynamic repertoire of a eukaryotic transcriptome surveyed at singlenucleotide resolution. Nature 453, 1239-1243 (2008). 
medRxiv preprint doi: https://doi.org/10.1101/2021.02.23.21252221; this version posted February 26, 2021. The copyright holder for this preprint

(which was not certified by peer review) is the author/funder, who has granted medRxiv a license to display the preprint in perpetuity.

All rights reserved. No reuse allowed without permission.

1468 94. Sultan, M., et al. A global view of gene activity and alternative splicing by deep sequencing of 1469 the human transcriptome. Science 321, 956-960 (2008).

1470 95. Tanabe, M. \& Kanehisa, M. Using the KEGG database resource. Curr Protoc Bioinformatics

$1471 \quad$ Chapter 1, Unit1 12 (2012).

1472 96. Kanehisa, M., Goto, S., Sato, Y., Furumichi, M. \& Tanabe, M. KEGG for integration and

1473 interpretation of large-scale molecular data sets. Nucleic Acids Res 40, D109-114 (2012).

1474 97. Law, C.W., et al. RNA-seq analysis is easy as 1-2-3 with limma, Glimma and edgeR. F1000Res

1475 5(2016).

1476 98. Law, C.W., Chen, Y., Shi, W. \& Smyth, G.K. voom: Precision weights unlock linear model analysis 1477 tools for RNA-seq read counts. Genome Biol 15, R29 (2014).

1478 99. Reshef, D.N., et al. Detecting novel associations in large data sets. Science 334, 1518-1524

1479 (2011).

1480 
Figure 1

Culture Positivity and risk for poor outcome $(n=589)$

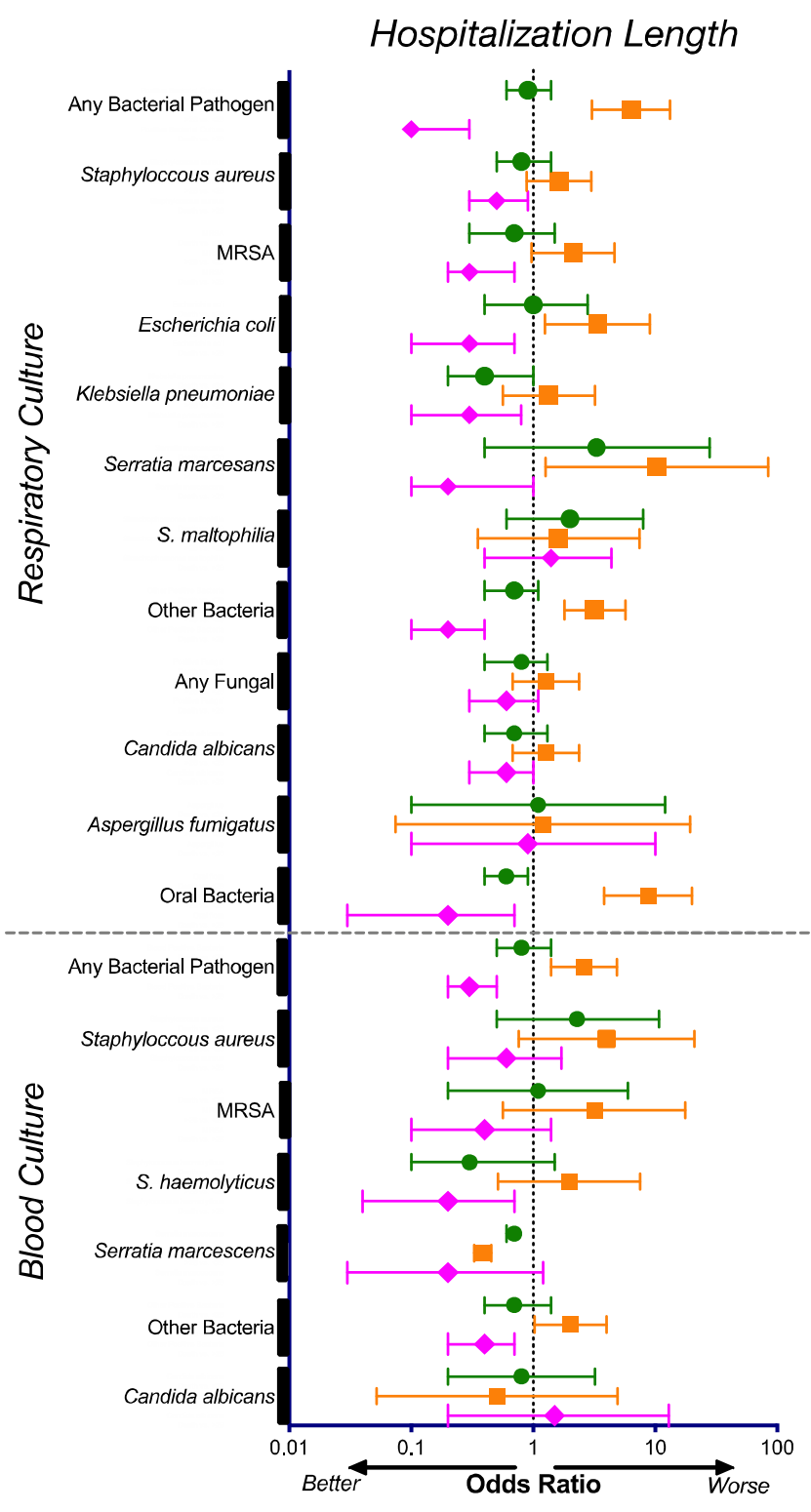

First 2 Weeks of Hospitalization

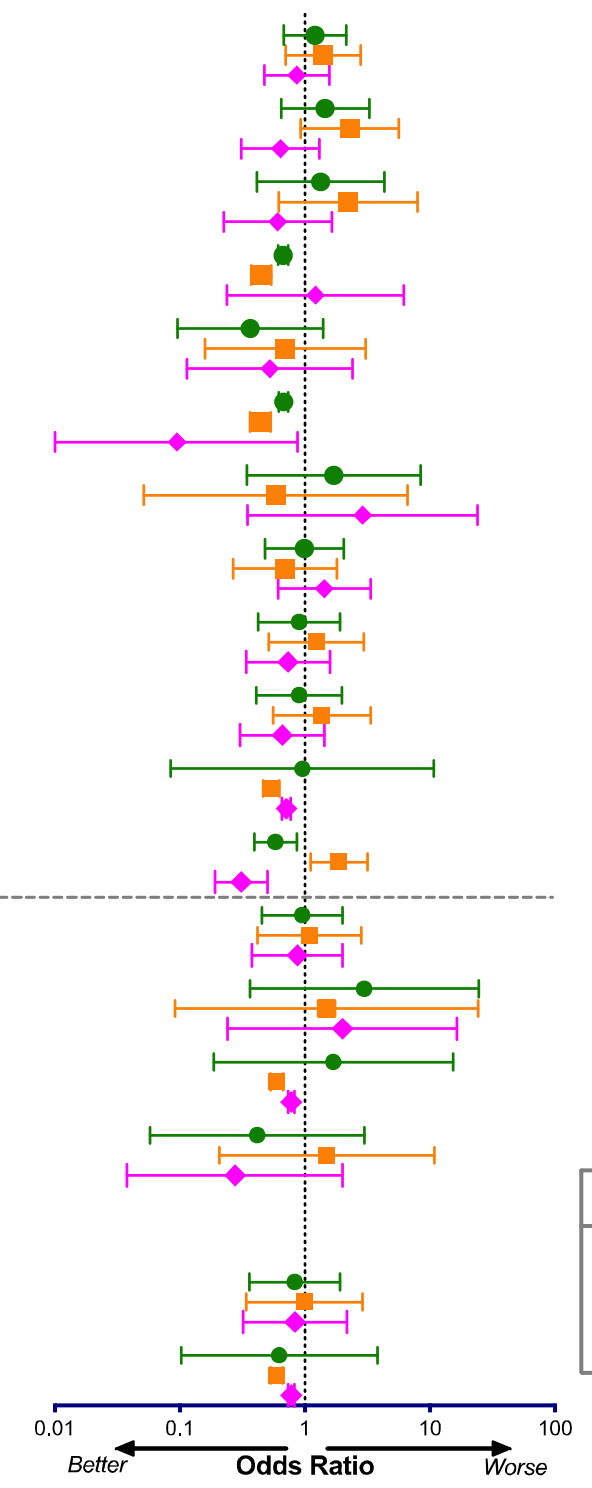

Figure 1. Associations between culture positivity and clinical outcome. Odds ratios and corresponding 95\% confidence intervals for rates of culture positivity for the whole cohort $(n=589)$ during the length of their hos pitalization (left) and during the first 2 weeks of hospitalization (right)

Outcome ris Better vs. Worse

Deceased vs. $\leq 28$ days

$>28$ days vs. $\leq 28$ days

Deceased vs. $>28$ days 
Figure 2

(a)

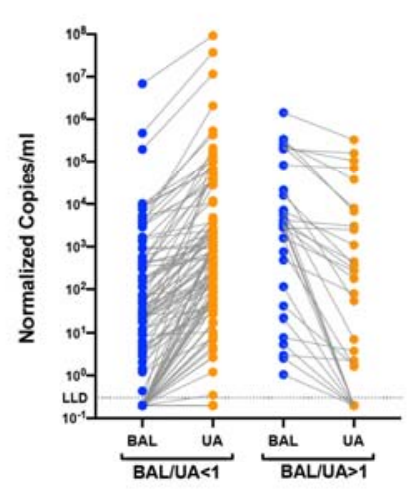

(b)

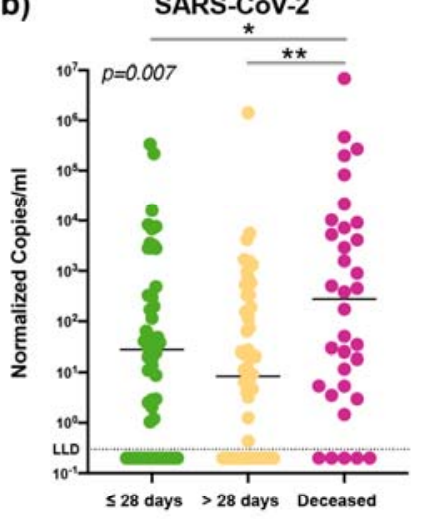

(c)

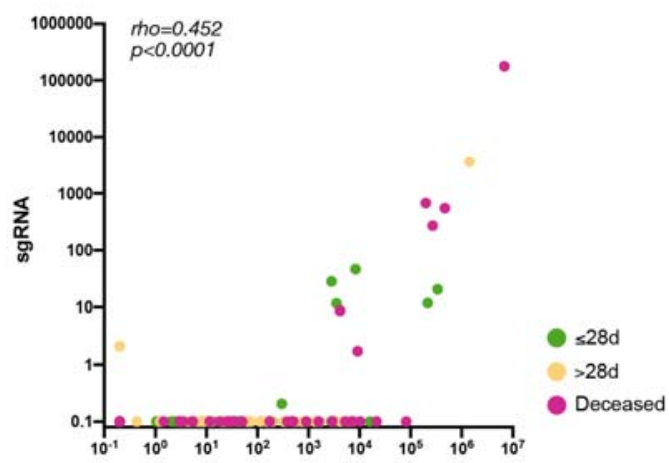

RT SARS_CoV_2 (Normalized Copies)

\section{(d) RNA virome}

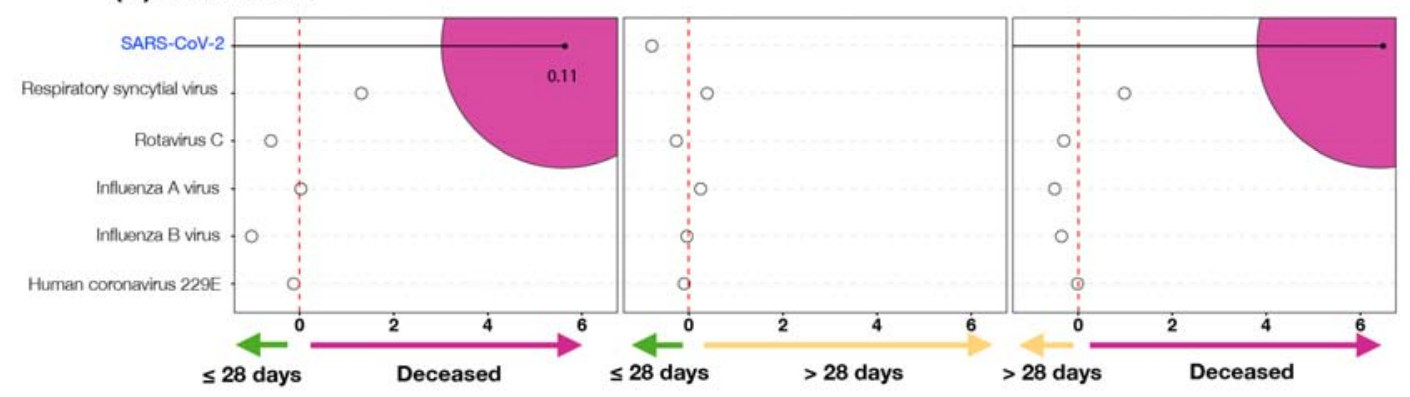

(e) DNA Phage metatranscriptome

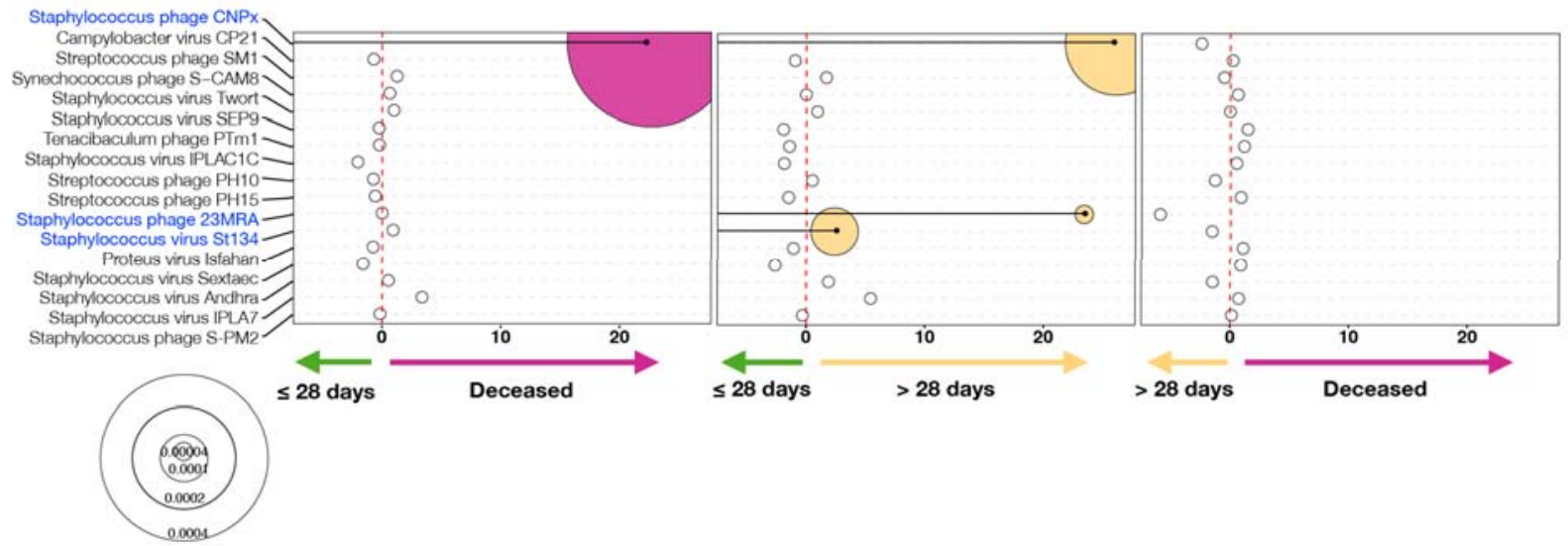

\section{Figure 2. SARS-CoV-2 viral load and virus}

metatranscriptome analyses. Copies of the SARS-CoV-

$2 \mathrm{~N}$ gene per $\mathrm{ml}$, normalized by the Human RNase $\mathrm{P}$ gene, comparing paired upper and lower airway samples (a) and levels in BAL comparing clinical outcome groups $(b, *=$ Mann-Whitney $U \mathrm{p}<0.05$ $* *=$ Mann-Whitney $U p<0.01$ ). (c) PCoA analysis based on Bray Curtis Dissimilarity index of BAL

Metatranscriptome data comparing clinical outcome (PERMANOVA p-value). Bubble plot showing DESeq results of RNA viruses (d) and expressed DNA phages (e) enriched in each clinical outcome comparisons (bubble size based on median relative abundance for those found statistically significant). 
(a)

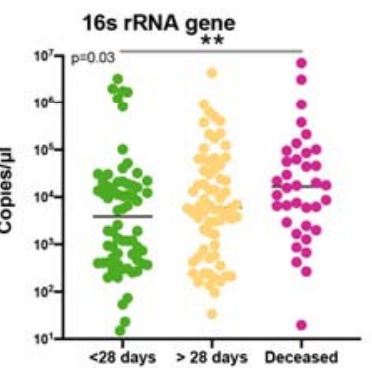

(b)

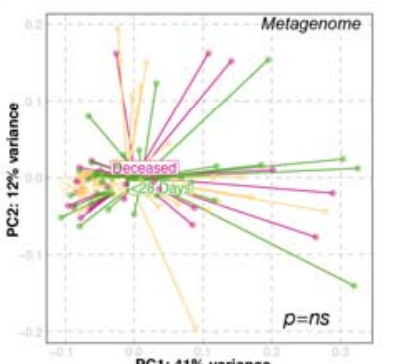

PC1: $41 \%$ variance (c)

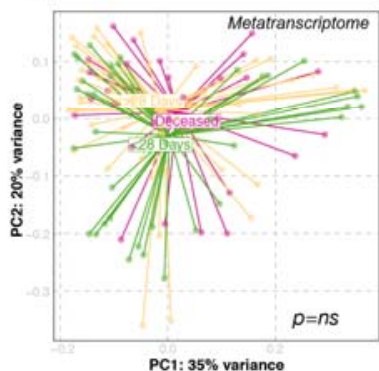

(d)

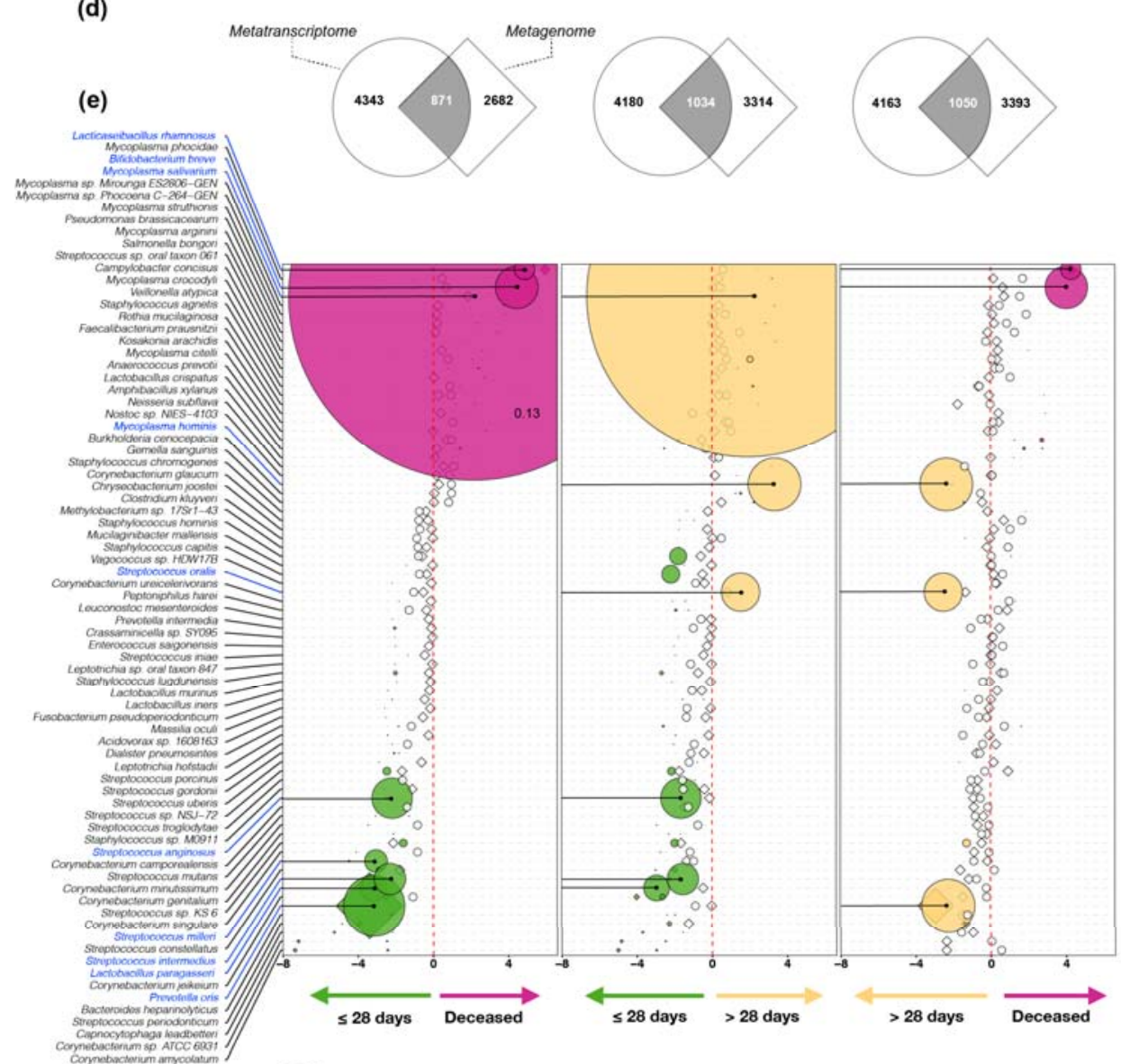

Figure 3. Bacteria load and taxonomic compositional analyses. (a)

Bacterial load measured by ddPCR targeting 165 rRNA gene ${ }^{* *}=$ Mann-

Whitney $U \mathrm{p}<0.01$ ). PCoA analysis based on Bray Curtis Dissimilarity index of BAL Metagenome (b) and Metatranscriptome (c) data comparing clinica outcome (PERMANOVA p-value). (d) Gene Set Enrichment Analysis (GSEA) was used to compare the taxonomic signatures identified in $\mathrm{BAL}$

metagenome (diamonds) and metatranscriptome (circles) as distinctly enriched for comparisons between clinical outcome groups (differential enrichment performed based on DESeq2 analysis). (e) Bubble plot showing DESeq results of bacteria found concordantly differentially enriched between clinical outcome groups (bubble size based on median relative abundance for those found statistically significant). 


\section{Figure 4}

(a)

(b)
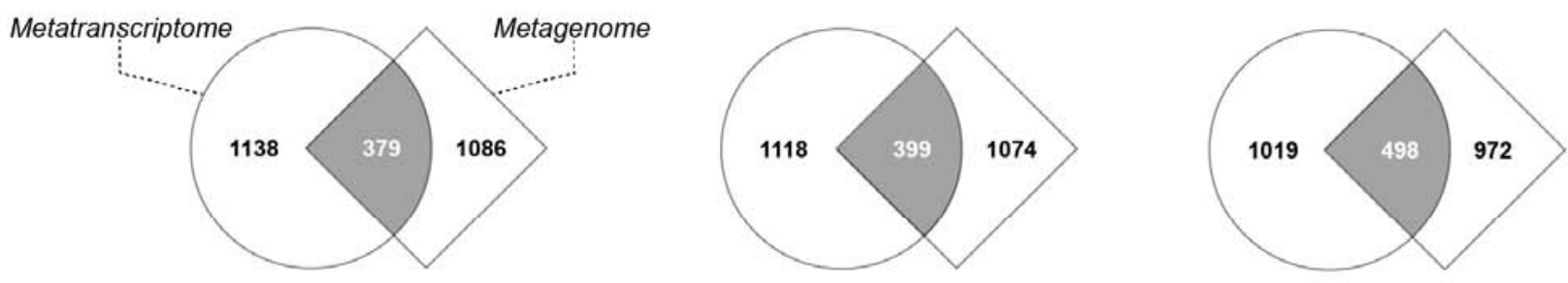

Vasopressin-regulated water reabsorption Microtubules mRNA processing factors Nitrate/nitrite transporters
Glycosphingolipid biosynthesis-ganglio series
Ascorbate and aldarate metabolism. Universal Cas proteins DNA Replication Initiation Factors MAPK signaling pathway - yeast Glycosaminoglycan degradation arious types of $\mathrm{N}$-glycan biosynthesis Various types of $\mathrm{N}$-glycan beriosynthesis
Transfering aldehyde or ketonic groups Glycosylases
Bacterial poylsaccharide

Oxidoreductase activity, acting on the $\mathrm{CH}-\mathrm{CH}$ group of donors AMPK signaling pathway Cytoskeletal proteins Lysosome Amino sugar and nucleotide sugar metabolism
Cell cycle yeast Transporters
Antimicrobial resistance genes Two-component system Aquaporins and small neutral solute transporters. Carbon-nitrogen lyases Monobactam biosynthesis Melanogenesis

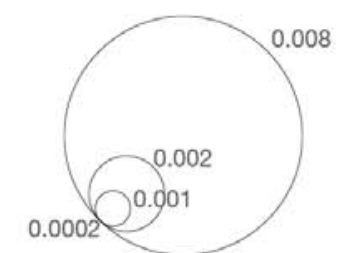

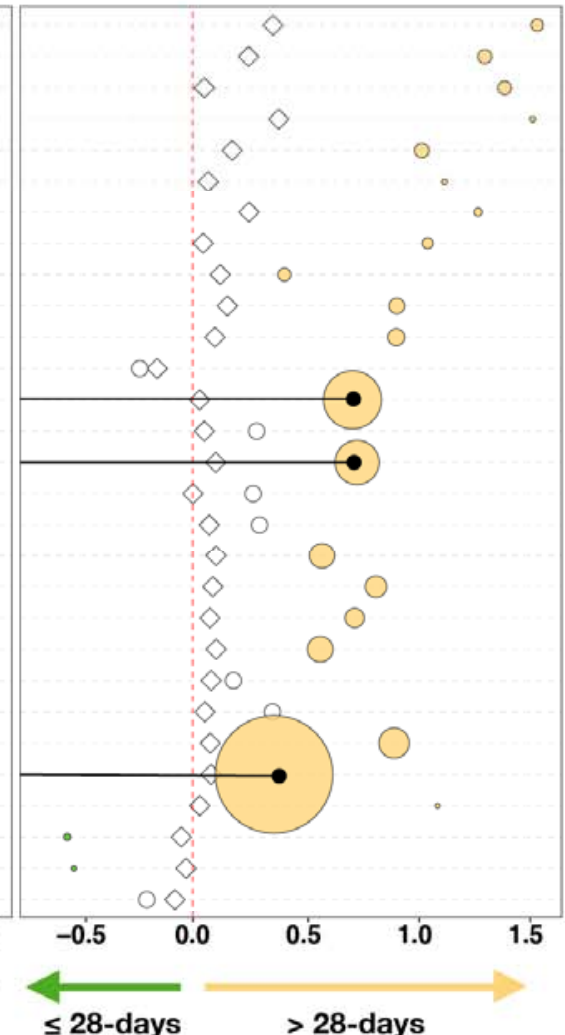

$\leq$ 28-days

Deceased

$\leq$ 28-days
$>$ 28-days

Figure 4. Functional microbial compositional analyses. KOs were summarized to associated pathways and differential expression was calculated based on DESeq 2 analysis. (a) Gene Set Enrichment Analysis (GSEA) was used to compare the functional signatures identified in BAL metagenome and metatranscriptome as distinctly enriched for comparisons between clinical outcome groups. (b) Bubble plot showing DESeq results of microbial functions found concordantly differentially enriched between clinical outcome

groups (bubble size based on median relative abundance for those found statistically significant). 


\section{Figure 5}

(a)

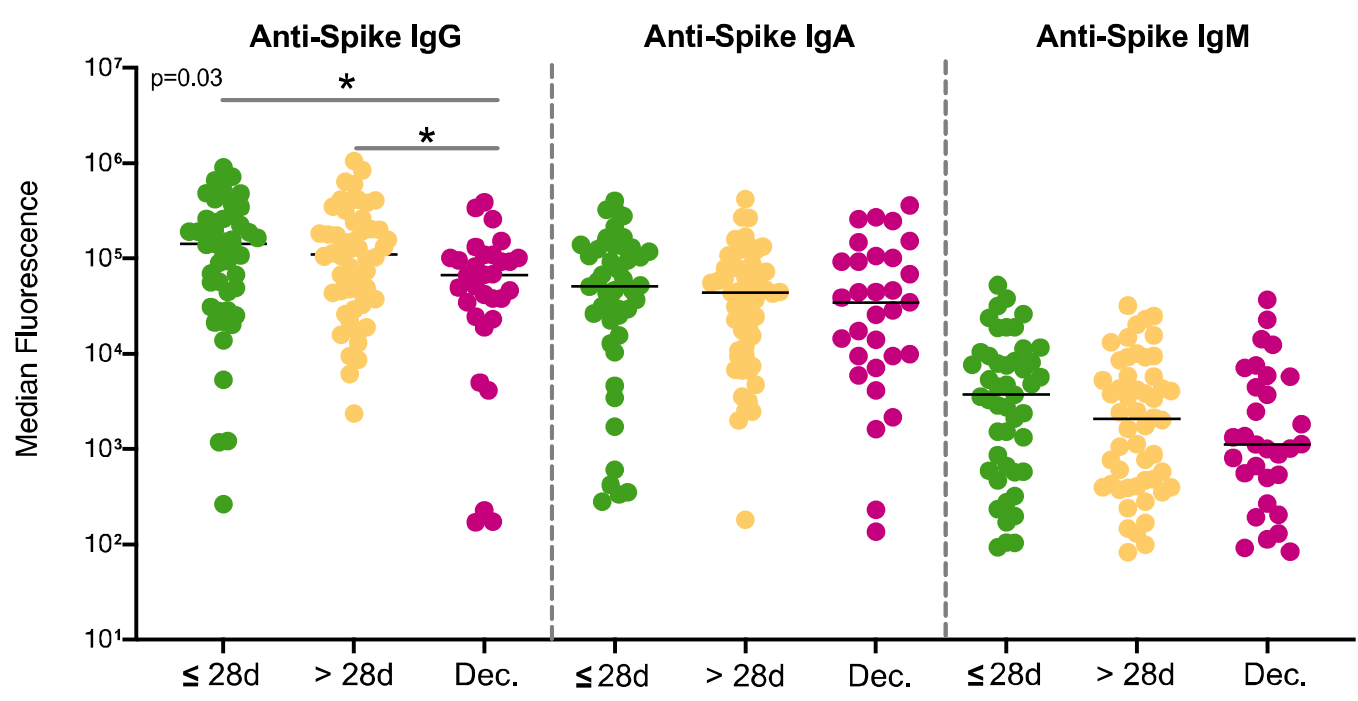

(c)

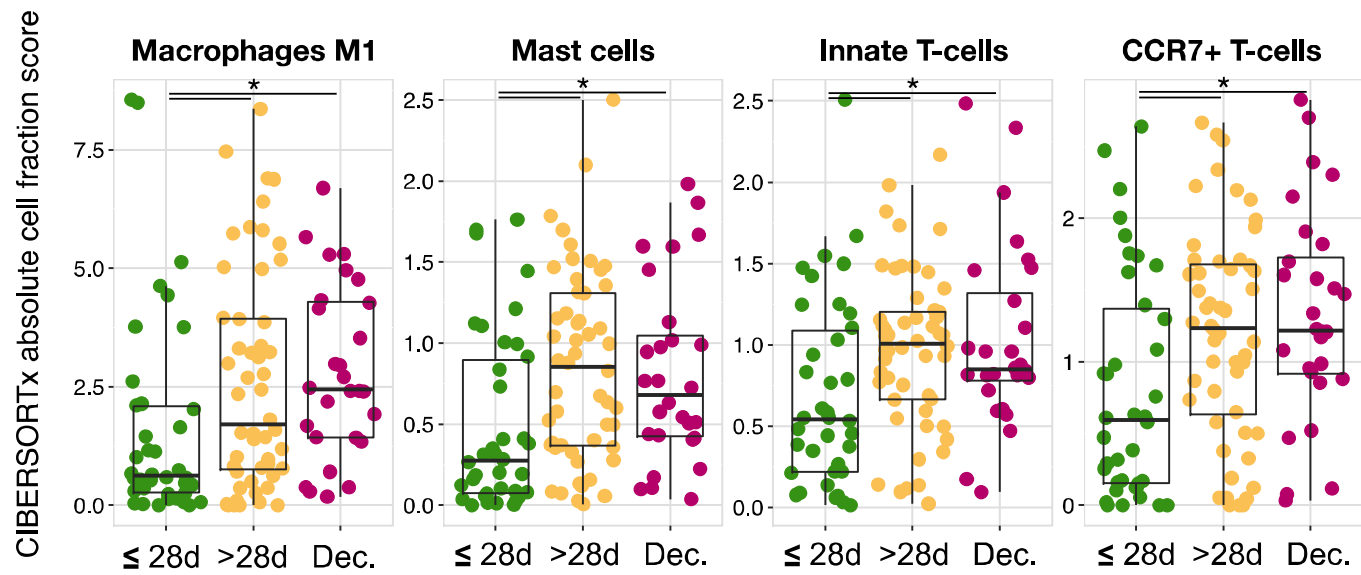

(b)

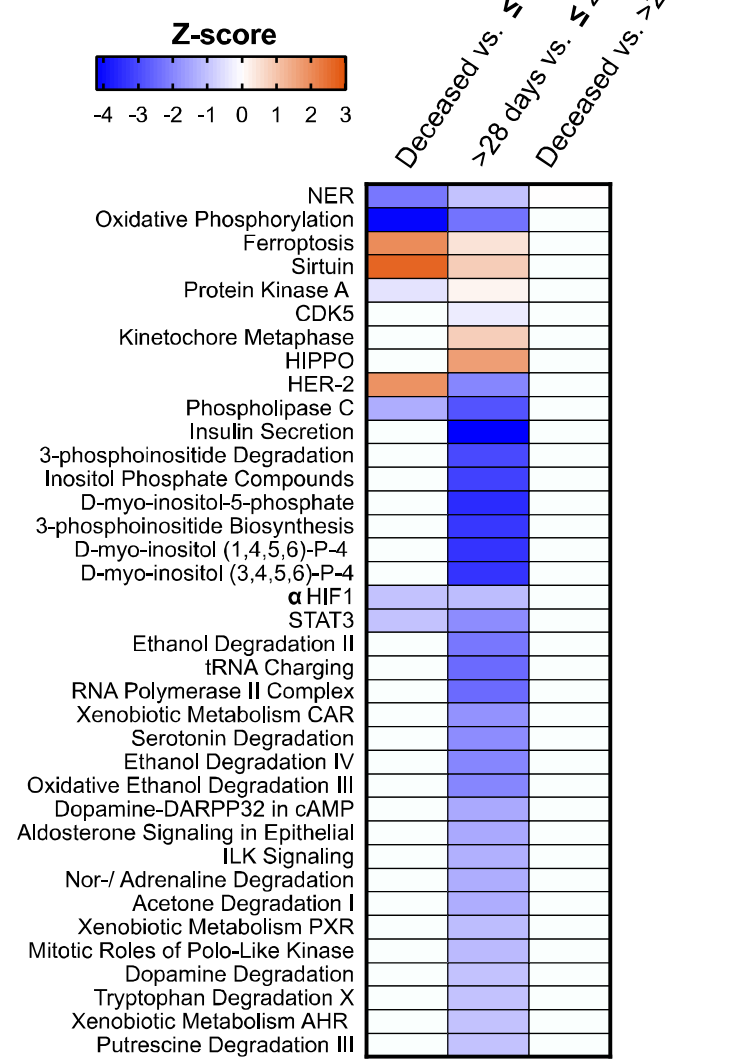

Figure 5. Lower airway host immune profiling in severely ill COVID-19. (a) Levels of anti-SARS-CoV-2 Spike antibodies in BAL $\left({ }^{*}=\right.$ Mann-Whitney $\left.\cup p<0.05\right)$. (b) Heat-map of canonical pathway analysis based on Ingenuity Pathway Analysis (IPA, RRID:SCR_008653) using the lower airway host transcriptome comparing clinical outcome groups. Orange shows up-regulation of pathway, blue shows down-regulation of pathway. (c) Cell type abundance quantification plots. Comparison of abundance of mast cells and neutrophils among outcome groups in the BAL fluids of critically ill patients with COVID-19. Cell type abundance was estimated from the host transcriptome with CIBERSORTX. Each dot denotes the quantification score of a sample and boxes depict median and inter-quartile range $\left({ }^{*}=\right.$ Mann-Whitney U $\mathrm{p}<0.05)$. 

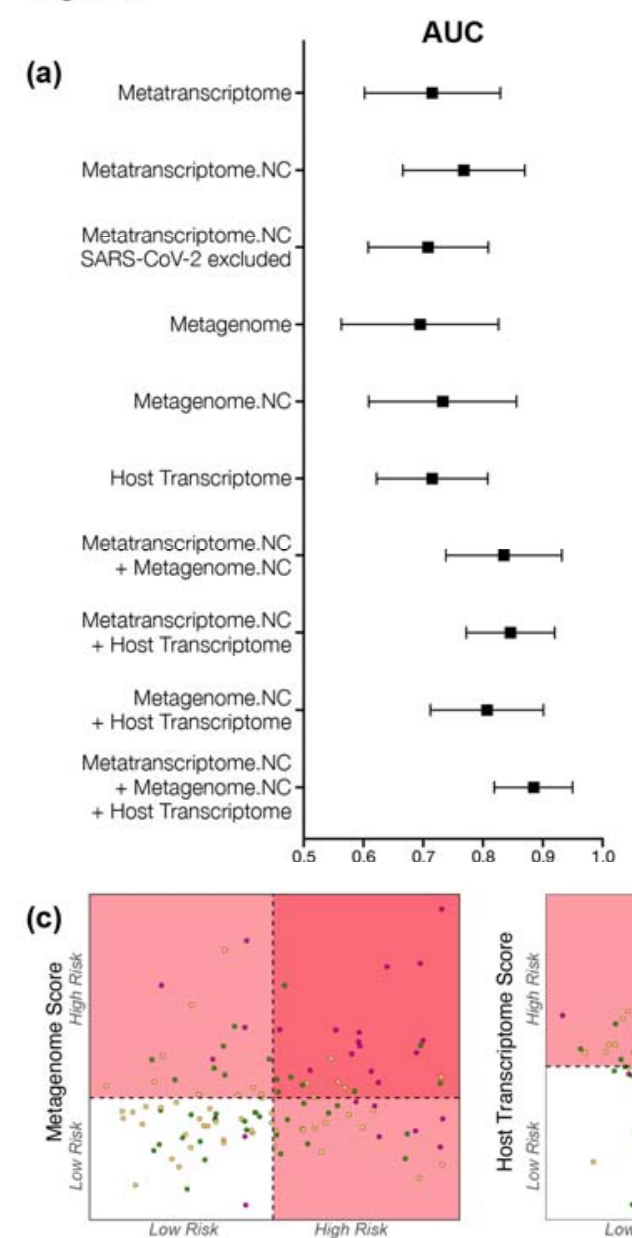

Low Risk High Risk

(d)

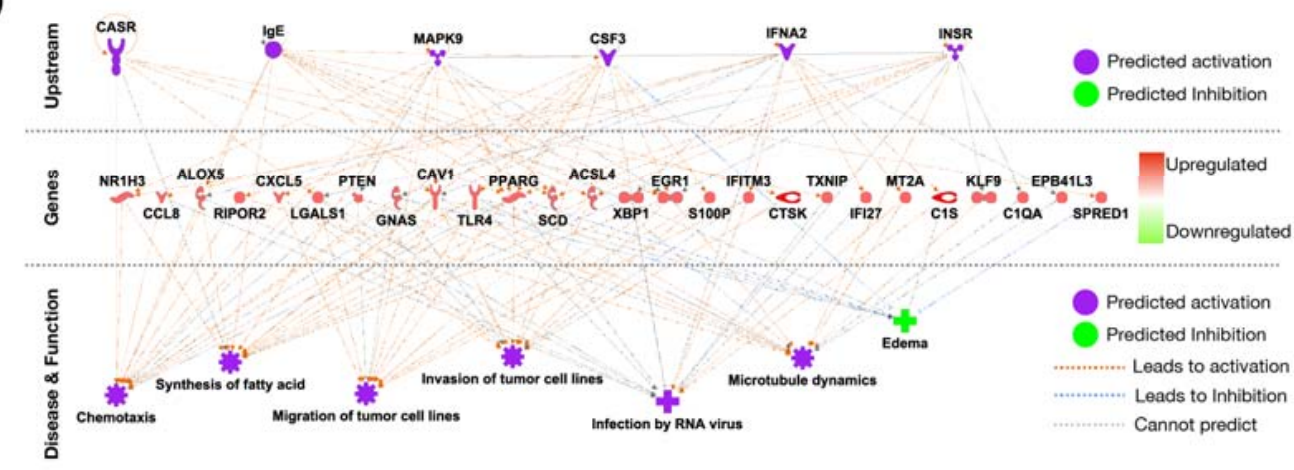

(b)
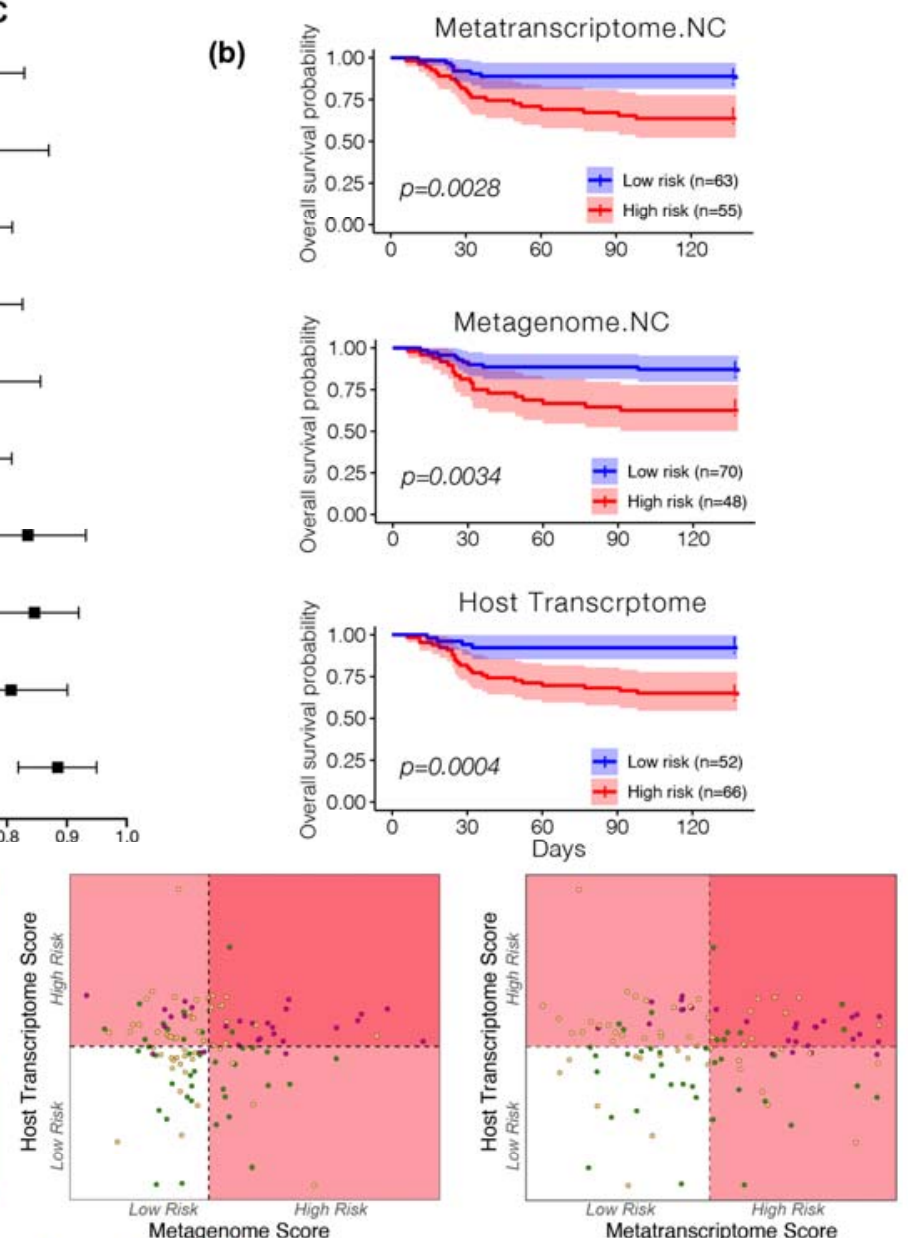

Metatranscriptome Score

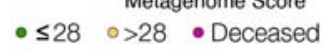

\section{Figure 6. Mortality predictive power of metatranscriptome,}

metagenome and host transcriptome. (a) Area under the curved median and confidence interval for receiver operating characteristic curve analyses calculated from each sequencing datasets as predictor and mortality as outcome. (b) Kaplan-meier survival analyses based on a cutoff value estimated from features selected from each sequencing dataset. The "High risk" and "Low risk" groups is the mean of predicted risk scores in all samples. (c) Scatterplot among risk scores from metatranscriptome, metagenome, and host transcriptome. Dotted line denotes the mean of the risk scores across all subjects, which is also the threshold for dividing the samples into "High risk" and "Low risk" groups. (d) IPA analyses of host transcriptomic signatures identified as most predictive of mortality. 